\title{
Light scalars with lepton number to solve the $(g-2)_{e}$ anomaly
}

\author{
Susan Gardner ${ }^{*}$ and Xinshuai Yan ${ }^{\dagger}$ \\ Department of Physics and Astronomy, University of Kentucky, Lexington, Kentucky 40506-0055 USA
}

(Received 19 August 2019; accepted 11 September 2020; published 14 October 2020)

\begin{abstract}
Scalars that carry lepton number can help mediate would-be lepton number-violating processes, such as neutrinoless double $\beta$ decay or lepton scattering-mediated nucleon-antinucleon conversion. Here, we show that such new scalars can also solve the anomaly in precision determinations of the fine-structure constant $\alpha$ from atom interferometry and from the electron's anomalous magnetic moment, $a_{e} \equiv(g-2)_{e} / 2$, by reducing $\left|a_{e}\right|$. Study of the phenomenological constraints on these solutions favor a doubly charged scalar with mass below the GeV scale. Significant constraints arise from the measurement of the parity-violating asymmetry in Møller scattering, and we consider the implications of the next-generation MOLLER experiment at Jefferson Laboratory and of an improved $a_{e}$ measurement.
\end{abstract}

DOI: 10.1103/PhysRevD.102.075016

\section{INTRODUCTION}

Through tour-de-force efforts in both theory and experiment, the anomalous magnetic moments of both the electron and muon have emerged as exquisitely sensitive probes of physics beyond the Standard Model (SM) [1-8]. For many years, the measured value of the electron's anomalous magnetic moment $a_{e} \equiv(g-2)_{e} / 2^{1}$ was used to determine the most precise value of the fine-structure constant $\alpha \equiv$ $e^{2} / 4 \pi \epsilon_{0} \hbar c$ [8], with the measurement of $a_{\mu}$ providing sensitivity to new physics at the weak scale, once the hadronic and electroweak contributions were taken into account $[6,10]$. In recent years, with the emergence of precise assessments of $a_{e}$ in QED perturbation theory through fifth order in $\alpha / \pi[1,11-14]$ and precise determinations of $\alpha[3,4]$ from atom interferometry [15,16], $a_{e}$ itself, due to its quantum nature, has also emerged as a probe of physics beyond the SM. Indeed, the comparison of $a_{e}$ from its direct experimental measurement with its expected value in the SM, using atom interferometry to fix $\alpha$, yields the most precise test of the SM in all of physics [17].

The SM value of $a_{e}$ is dominated by the contribution from QED — though contributions from the SM weak gauge

*gardner@pa.uky.edu

Present address: Institute of Particle Physics and Key Laboratory of Quark and Lepton Physics (MOE), Central China Normal University, Wuhan, Hubei 430079, China.

xinshuai@ccnu.edu.cn

${ }^{1}$ In this paper, we define the magnetic moment of a charged lepton $\ell$ as $\boldsymbol{\mu}_{\ell}=g_{\ell} \mathbf{S} e / 2 m_{\ell}$, with $g_{\ell}>0$ and $e=-|e|$ [9].

Published by the American Physical Society under the terms of the Creative Commons Attribution 4.0 International license. Further distribution of this work must maintain attribution to the author(s) and the published article's title, journal citation, and DOI. Funded by SCOAP ${ }^{3}$. bosons $W^{ \pm}, Z^{0}$ and hadronic effects also exist, these are known to be extraordinarily small, contributing only 0.026 [18-20] and $1.47 \mathrm{ppb}$ [21,22], respectively, of the total contribution to $a_{e}^{\mathrm{SM}}$ [1]. The analysis of atom interferometry measurements for $\alpha$ also require the use of QED theory and other observables [8], though the uncertainty in the determined value of $\alpha$ is dominated by that in its measured observable, $h / M_{X}$, where $h$ is Planck's constant and $M_{X}$ is the mass of atomic species $X$. With the most precise experimental result for $a_{e}[2,23]$ and $h / M_{X}$ measurements for $\mathrm{Rb}$ [3] or Cs atoms [4] to determine $\alpha$ and thus $a_{e}^{\mathrm{SM}}$ [1], we report [1]

$$
\begin{gathered}
a_{e}^{\mathrm{EXP}}-a_{e}^{\mathrm{SM}}[\mathrm{Rb}]=(-131 \pm 77) \times 10^{-14}, \\
a_{e}^{\mathrm{EXP}}-a_{e}^{\mathrm{SM}}[\mathrm{Cs}]=(-88 \pm 36) \times 10^{-14},
\end{gathered}
$$

where here and elsewhere the uncertainties are added in quadrature. In what follows, we use the most precise determination of $\alpha$ to define the $a_{e}$ anomaly, $\Delta a_{e} \equiv$ $(-88 \pm 36) \times 10^{-14}[1,2,4]$, which is a discrepancy of approximately $2.4 \sigma$. For reference, we report the anomaly in $(g-2)_{\mu}$ as well $[5,24]$,

$$
\Delta a_{\mu} \equiv a_{\mu}^{\mathrm{EXP}}-a_{\mu}^{\mathrm{SM}}=(2.74 \pm 0.73) \times 10^{-9},
$$

for a discrepancy of approximately $3.7 \sigma$, as also determined by Ref. [25], with a sign opposite to that of $\Delta a_{e}$. Both the relative sign and size of the anomalies suggest distinct mechanisms for their explanation. For example, if weakscale new physics were to explain $\Delta a_{\mu}$, scaling as $m_{\mu}^{2}$, then its contribution to $\Delta a_{e}$ would be roughly ten times too small, $\Delta a_{e} \simeq 0.7 \times 10^{-13}$ [26,27]. Thus, explaining both anomalies is seemingly not possible in models that 
differentiate electrons and muons only by their massrather, possible solutions should break lepton flavor universality [27-30]. The relatively large size of $\Delta a_{e}$ also suggests the appearance of new physics below approximately the $1 \mathrm{GeV}$ scale.

Several models of light new physics have been proposed that could explain both of the $a_{\ell}$ anomalies [27-30]. However, the suggestion that new physics at scales below approximately $1 \mathrm{GeV}$, arising from so-called dark, hidden, or secluded sectors, could explain the $a_{\mu}$ anomaly has existed much longer [31,32]. Keen interest in such scenarios has been generated not only by anomalies in highenergy astrophysics that could arise from dark-matter annihilation [33] but also by an appreciation of the great reaches of untested parameter space possible for their realization [34,35], which has spurred new experimental initiatives [36,37]. Although the possibility that a U(1) gauge boson that mixes with the photon [38], a "dark photon," could explain $\Delta a_{\mu}$ has been ruled out [39], solutions involving a new light scalar or pseudoscalar are still possible [40,41]. Since the dark photon gives a positive contribution to $a_{\ell}$ [31,32], it also cannot address the $a_{e}$ anomaly [4].

Models that address both $a_{\ell}$ anomalies treat the electrons and muons in different ways. In Ref. [28], a single real scalar is introduced, and, in the electron case, the scalar coupling to a heavy charged fermion, such as the $\tau$, can be chosen to mediate a two-loop Barr-Zee [42] contribution to $a_{e}$ that yields the needed opposite sign. In Ref. [29], models with an Abelian flavor symmetry $\mathrm{L}_{\mu}-\mathrm{L}_{\tau}$ are used to realize different contributions to $a_{e}$ and $a_{\mu}$, with the suggested consequence that the permanent electric-dipole moment (EDM) of the $\mu$ could be much larger than supposed from electron EDM limits. In Ref. [27], a complex scalar is introduced with $C P$-odd couplings to the electron and $C P$-even couplings to the muon, generating contributions to $a_{e, \mu}$ of opposite sign. The somewhat disjoint nature of the various simultaneous solutions, and the severity of the constraint from nonobservation of $\mu \rightarrow$ er [29], suggests that we can address one anomaly without precluding the other. In this paper, we show that we can solve the $a_{e}$ anomaly by introducing a scalar with lepton number that couples to first-generation fermions only, respecting SM symmetries, supposing that one of the solutions for $\Delta a_{\mu}$ proposed in Refs. [27,40,41,43-46], e.g., could also act. The solutions we have found also serve as ingredients in minimal scalar models [47-51] that can also mediate lepton number-violating processes, such as neutrinoless double $\beta$ decay and scattering-mediated nucleon-antinucleon conversion [51].

Giudice et al. have shown that many possible new physics models could generate a shift of $a_{e}$ from its SM value [26], considering both models that connect to a change in $a_{\mu}$ by $\left(m_{\mu} / m_{e}\right)^{2}$ and those that do not. In the latter class, they consider models that connect to violations of charged lepton flavor number or lepton flavor universality, as well as models with heavy vectorlike fermions $[26,52]$. In the last example, Giudice et al. introduced a $\mathrm{SU}(2)$ vectorlike doublet and singlet, with interactions that can explicitly break lepton number. In what follows, we consider a new physics model for $\Delta a_{e}$ of a completely different kind-here the scalars carry lepton number, with scalar-fermion interactions that conserve lepton number, and indeed are SM-gauge invariant, and these features are essential to the results we find. Other models pertinent to $a_{e}$ $[53,54]$ that also address the $\Delta a_{e}$ anomaly [54] have been proposed. Interestingly, models with a new axial-vector boson also generate contributions that decrease $\left|a_{e}\right|$ $[31,55]$, though other empirical constraints exist on these solutions as well [4,55].

Scalars that carry lepton number also appear in neutrino mass models. Although the smallness of the neutrino masses can be elegantly ascribed to a seesaw mechanism with a new physics scale of some $M_{\mathrm{N}} \sim 10^{10-15} \mathrm{GeV}$ [56-59], there are many alternate possibilities [60]. In type II seesaw models [61-65], e.g., the seesaw scale, can be below the electroweak scale. The neutrino masses can also be generated radiatively [64,66-73]. The scalars of interest to us appear in different contexts. For example, weak-isospin singlet scalars appear in radiative mass models [67,68,72], whereas weak-isospin triplet scalars appear in light type II seesaw models and other mass models [74]. If the scalar also couples to right-handed $W^{ \pm}$ gauge bosons, as in the latter case in the left-right symmetric model, the scalar-fermion coupling for a scalar that couples to right-handed electrons with a scalar mass of less than approximately $100 \mathrm{GeV}$ is significantly constrained by existing experimental limits on neutrinoless double $\beta$ decay [74]. This constraint does not act in our case because the associated scalars do not break lepton number. Here, we suppose, as in Ref. [51], that scalars with lepton number need not in themselves act to explain the numerical size of the neutrino mass, so that we take no stance on the precise origin of the neutrino masses and mixings. We consider minimal scalar models with weak-isospin triplet and singlet scalars that couple to first-generation fermions only-such a scenario is much less constrained, evading severe constraints, e.g., from the $\mu$ lifetime and $\mu \rightarrow e \gamma$ decay [72]. We do find constraints, however, on our scenario from precision measurements of Bhabha scattering and of the $Z^{0}$ width. We view minimal scalar models as a simple framework in which to study the connections between B- and L-violating phenomena, and for scalars with masses that would permit contributions to the $Z^{0}$ width, we find that it turns out to be incomplete. We also find, however, that it is simple to remedy this and bring all into agreement through the addition of a higher dimensional operator, and its impact on the parameters of our solutions to the $\Delta a_{e}$ anomaly is trivially small. We consider these issues in Sec. V. 
We conclude this section by outlining the content of our paper. We begin, in Sec. II, by describing the scalar models we employ in more detail. Thereafter, in Sec. III, we discuss the contributions to $a_{e}$ in these models, providing our detailed computations in the Appendix for clarity. We describe the sets of possible couplings and masses that solve the $a_{e}$ anomaly before turning to the constraints on these models from parity-violating electron scattering in Sec. IV and considering other possible constraints in Sec. V. In our analysis, we focus on scalars of less than $\mathcal{O}(10 \mathrm{GeV})$ in mass, making our analysis complementary to that of Ref. [74], which analyzed constraints on doubly charged scalars with masses in excess of that. We conclude with a discussion of the experimental prospects in Sec. VI.

\section{SCALARS WITH LEPTON NUMBER}

Minimal scalar models are extensions of the SM that respect its gauge symmetries and do not impact its predictive power because the new interactions possess mass dimension 4 or less. Such models have been primarily employed in the study of baryon number-violating and/or lepton number-violating processes [47-51,75], through the low-energy higher-dimension operators that can appear. In what follows, we introduce new scalars with definite representations under the $\mathrm{SU}(3)_{c} \times \mathrm{SU}(2)_{L} \times U(1)_{Y}$ gauge symmetry of the SM that also carry nonzero lepton number $\mathrm{L}$ and construct their minimal interactions by requiring Lorentz and SM gauge invariance. Scalars that carry baryon number appear in this model also, and the possiblity of baryon number-violating proton or neutron decay is removed at tree level by choosing the particular scalars that are allowed to appear [49-51]. In such an approach, the observability of the baryon number-violating and/or lepton number-violating processes that can occur rest on the empirical constraints that exist on the new scalars' masses and couplings [51]. This is in contrast to UV-complete models in which the gauge dynamics enforce the absence of baryon-number violation by one unit but also admit observable neutron-antineutron oscillations. For specific examples, we note models based on the gauge group $\mathrm{SU}(3)_{c} \times \mathrm{SU}(2)_{L} \times \mathrm{SU}(2)_{R} \times \mathrm{U}(1)_{\mathrm{B}-\mathrm{L}}[76,77]$ or $\mathrm{SU}(2)_{L} \times \mathrm{SU}(2)_{R} \times \mathrm{SU}(4)_{c}$ [78,79] or on the nonsupersymmetric $\mathrm{SO}(10)$ [80]. In these models, the new light scalars range from about $100 \mathrm{GeV}$ to the $\mathrm{TeV}$ scale in mass. Thus, minimal scalar models open the door to new possibilities, to the consideration of a relatively unexplored parameter space. In this paper, we show that new sub-GeVscale scalars can potentially explain the $(g-2)_{e}$ puzzle, but to render these solutions compatible with measurements from the Large Electron-Positron Collider (LEP) we need to augment our minimal scalar model with a higherdimension operator. We refer to Sec. V for a detailed discussion.

Generally, there are three possible scalars $X_{i}$ that couple to SM leptons only, all carrying $\mathrm{L}=-2$. We have two weak-isospin singlets: $X_{1}$ with hypercharge $Y=2$ that couples to right-handed fermions, where we employ the convention that the electric charge $Q_{\mathrm{em}}=T_{3}+Y$ in units of $|e|$ and $T_{3}$ is the third component of weak isospin, and $X_{2}$ with hypercharge $Y=1$ that couples to left-handed fermions. There is also one weak-isospin triplet $X_{3}$ with $Y=1$ that couples to left-handed fermions. Since the new scalars carry electric charge to ensure electric charge conservation, we have added scalar QED interactions as appropriate. Through the electroweak gauge invariant kinetic terms, the scalars couple to the $Z^{0}$ gauge boson as well, and we consider the consequences of this in Sec. V. We will see that our solutions to the $(g-2)_{e}$ puzzle demand scalars that are lighter than the $Z^{0}$ width constraints would allow, but we find that through a small addition to our minimal scalar models we can satisfy the $Z^{0}$ width constraint with only trivial numerical modifications to our $(g-2)_{e}$ solutions.

Denoting a right-handed lepton of generation $a$ as $e^{a}$ and the associated left-handed lepton doublet as $L^{a}$, the possible scalar-fermion interactions mediated by each $X_{i}$ are of the form

$-g_{1}^{a b} X_{1}\left(e^{a} e^{b}\right), \quad-g_{2}^{a b} X_{2}\left(L^{a} \varepsilon L^{b}\right), \quad-g_{3}^{a b} X_{3}^{A}\left(L^{a} \xi^{A} L^{b}\right)$,

where $\varepsilon=i \tau^{2}$ is a totally antisymmetric tensor, $\xi^{A} \equiv$ $\left(\left(1+\tau^{3}\right) / 2, \tau^{1} / \sqrt{2},\left(1-\tau^{3}\right) / 2\right)$, and $\tau^{A}$ are Pauli matrices with $A \in 1,2,3$ [51]. The symmetries of the scalar representations under weak-isospin $\mathrm{SU}(2)$ fix the symmetry of the associated coupling constant under $a, b$ interchange, with $g_{1}^{a b}$ and $g_{3}^{a b}$ symmetric and $g_{2}^{a b}$ antisymmetric. Thus, only $X_{1}$ and $X_{3}$ can couple to first-generation leptons exclusively. In Eq. (4), we adopt 2-spinors such that the fermion products in parentheses are Lorentz invariant, and we map to 4 -spinors via $\left(e_{L, R \alpha} \mu_{L, R \beta}\right) \rightarrow\left(e_{\alpha}^{T} C P_{L, R} \mu_{\beta}\right)$, where $C$ and $P_{L, R}=\left(1 \mp \gamma_{5}\right) / 2$ are in Weyl representation [81]. We have chosen the arbitrary phases [82] that appear such that $C=i \gamma^{0} \gamma^{2}$ and the charge-conjugate field $\psi^{c}$ is $\psi^{c} \equiv C(\bar{\psi})^{\top}$. Thus, the scalar-fermion interactions for each of these scalars are of the form

$$
\begin{aligned}
\mathcal{L}_{X_{1}} \supset & -g_{1}^{a b} X_{1} \overline{e_{R}^{a c}} e_{R}^{b}+\text { H.c., } \\
\mathcal{L}_{X_{2}} \supset & -g_{2}^{a b} X_{2}\left(\overline{e_{L}^{a c}} \nu_{L}^{b}-\overline{e_{L}^{b c}} \nu_{L}^{a}\right)+\text { H.c. } \\
\mathcal{L}_{X_{3}} \supset & -g_{3}^{a b}\left(X_{3}^{1} \overline{\nu_{L}^{a c}} \nu_{L}^{b}+X_{3}^{2} \frac{1}{\sqrt{2}}\left(\overline{e_{L}^{a c}} \nu_{L}^{b}+\overline{\nu_{L}^{a c}} e_{L}^{b}\right)\right. \\
& \left.+X_{3}^{3} \overline{e_{L}^{a c}} e_{L}^{b}\right)+ \text { H.c. }
\end{aligned}
$$

In what follows, we assume that $X_{1}$ and $X_{3}$ couple to firstgeneration fermions only, whereas for $X_{2}$, we assume only $1 \leftrightarrow 3$ couplings exist, since the existing constraints on intergenerational mixing are less severe in that case [72]. We analyze the pertinent constraints there in Sec. V. 


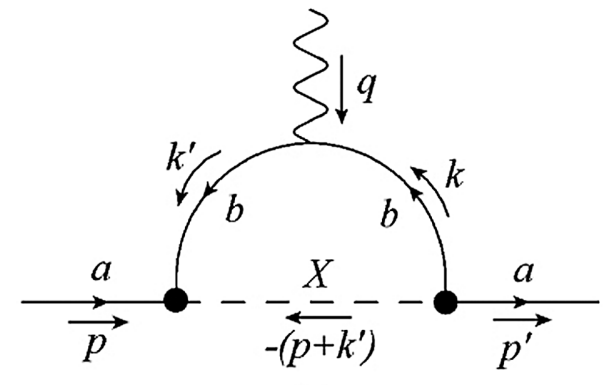

(a)

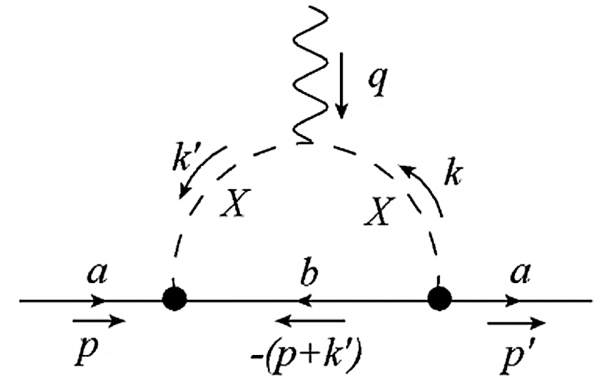

(b)

FIG. 1. Feynman diagrams to illustrate contributions to the anomalous magnetic dipole moment $a_{\ell_{a}}$ of lepton $a$, where $b$ denotes another lepton and $X$ denotes a scalar that carries lepton number. Note that if lepton $\mathrm{b}$ is electrically neutral, only diagram (b) can contribute to $a_{\ell_{a}}$.

\section{NEW SCALAR CONTRIBUTIONS TO $a_{e}$}

In minimal scalar models, the new scalars can carry electric charge, so that two types of Feynman diagrams can contribute to $a_{e}$ at leading order: one in which the photon attaches to the internal charged fermion line and a second in which the photon attaches to the charged scalar line-we illustrate these possibilities in Fig. 1. We find that $X_{1}$ and $X_{3}$ can contribute to $a_{e}$ through both diagrams, whereas in the case of $X_{2}$, only the second diagram appears. The contributions to $a_{e}$ from $X_{1}$ and $X_{2}$ have been previously studied [72]. Although we agree with Ref. [72] for the computation of $\Delta a_{e}$ from $X_{2}$, our computation of $\Delta a_{e}$ from $X_{1}$ does not-indeed, our result differs from the results of that reference by a factor of -4 . Consequently, we find that the contribution to $\Delta a_{e}$ from each scalar is negative definite. Since this result is key to our paper, and subtleties exist in the computation of $\Delta a_{e}$, we present our computation in detail in the Appendix. In this section, we compile our results and evaluate their consequences. We evaluate the contribution of each possible new scalar to $a_{e}$ independently, terming this $\left(\delta a_{e}\right)_{X_{i}}$.

Combining the results of the Appendix, Eqs. (A26) and (A34), as appropriate, we find that the contribution to $\Delta a_{e}$ from $X_{1}$ is

$$
\begin{aligned}
\left(\delta a_{e}\right)_{X_{1}}= & -\frac{m_{e}^{2}\left|g_{1}^{11}\right|^{2}}{4 \pi^{2}}\left(\int_{0}^{1} d z \frac{z(1-z)^{2}}{(1-z)^{2} m_{e}^{2}+z m_{X_{1}}^{2}}\right. \\
& \left.+2 \int_{0}^{1} d z \frac{z(1-z)^{2}}{z^{2} m_{e}^{2}+(1-z) m_{X_{1}}^{2}}\right)
\end{aligned}
$$

thus $\left(\delta a_{e}\right)_{X_{1}} \leq 0$ and finite for all $m_{X_{1}}>0$. Moreover, the contribution to $\Delta a_{e}$ from $X_{2}$ from Eq. (A35) is

$$
\left(\delta a_{e}\right)_{X_{2}}=-\frac{4 m_{e}^{2}\left|g_{2}^{1 j}\right|^{2}}{16 \pi^{2}}\left(\int_{0}^{1} d z \frac{z(1-z)}{m_{X_{2}}^{2}-z m_{e}^{2}}\right),
$$

where we have set the mass of the neutrino $\nu_{j}$ to zero here and elsewhere, as it is known to be very small [7]. The 4 in the numerator appears because $g_{2}^{13}=-g_{2}^{31}$, as in Ref. [72], so that there is a 2 in the effective $e-X_{2}-\nu_{j}$ vertex. Here, $M_{X_{2}}<m_{e}$ leads to a singularity in the parameter integral arising from on-mass-shell intermediate states; we avoid this possibility if $M_{X_{2}}>m_{e}$. For $M_{X_{2}}<m_{e}$, we would replace the integral in Eq. (7) with its principal value, though in that region, $\left(\delta a_{e}\right)_{X_{2}}>0$. Finally, the contribution to $\Delta a_{e}$ from $X_{3}$ is

$$
\begin{aligned}
\left(\delta a_{e}\right)_{X_{3}}= & -\frac{m_{e}^{2}\left|g_{3}^{11}\right|^{2}}{4 \pi^{2}}\left(\int_{0}^{1} d z \frac{z(1-z)^{2}}{(1-z)^{2} m_{e}^{2}+z m_{X_{3}}^{2}}\right. \\
& +2 \int_{0}^{1} d z \frac{z(1-z)^{2}}{z^{2} m_{e}^{2}+(1-z) m_{X_{3}}^{2}} \\
& \left.+\frac{1}{2} \int_{0}^{1} d z \frac{z(1-z)}{m_{X_{3}}^{2}-z m_{e}^{2}}\right) .
\end{aligned}
$$

Here, too, by choosing $M_{X_{3}}>m_{e}$, we would avoid the inconvenience of a singularity in the parameter integral; in the $M_{X_{3}}<m_{e}$ region, the integral would be replaced by its principal value, noting that in this case $\left(\delta a_{e}\right)_{X_{3}}<0$ for $M_{X_{3}}>0$. Thus, we observe that each of the three lepton number-carrying scalars possible in minimal scalar models could solve the $a_{e}$ anomaly - we need only choose a scalar mass and scalar-fermion coupling consistent with the empirical value of $\Delta a_{e}$, and a broad range of choices is possible. Thus, we see that the $\Delta a_{e}$ anomaly could also potentially be solved by new physics at very light mass scales, beyond the reach of existing accelerator experiments. Nevertheless, in what follows, we consider scalars with masses $M_{X_{i}}>m_{e}$, as that mass region loosely avoids astrophysical constraints, such as those from stellar cooling [83]. We note, however, that new particles with masses $M_{X_{i}}<m_{e}$ may be possible if their interactions do not permit them to escape an astrophysical environment [84] and our lepton number-carrying scalars may well be of that class. We also consider $M_{X_{i}}<8 \mathrm{GeV}$ on $X_{1}$ and $X_{3}$ because we note that existing LHC searches for new 


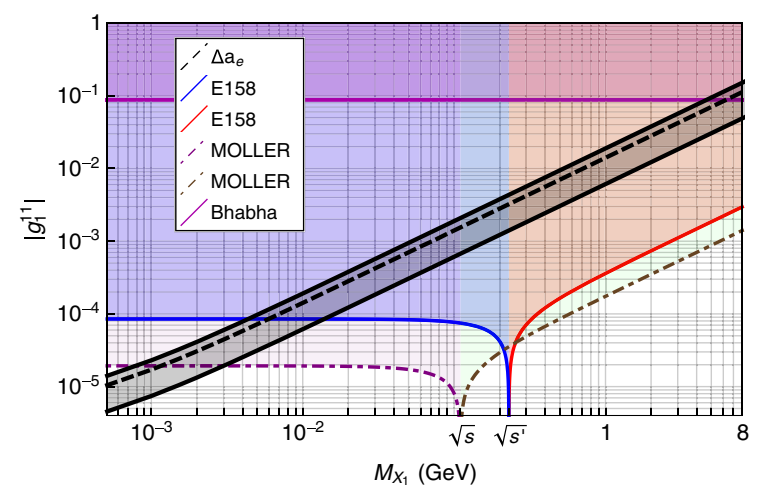

(a)

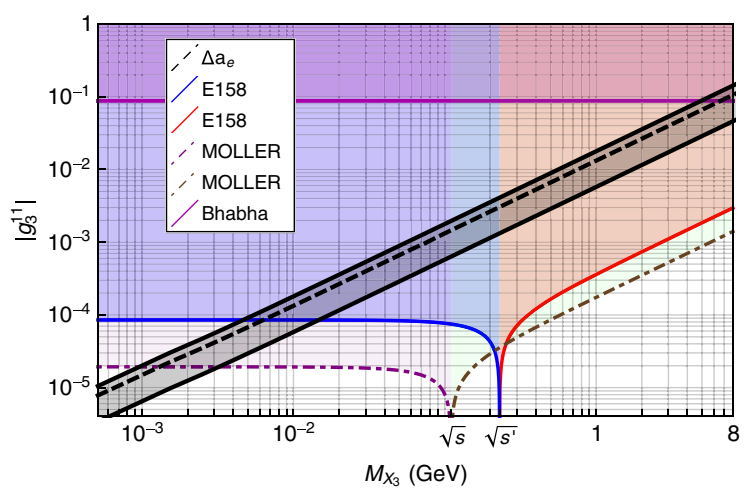

(b)

FIG. 2. Our solution for the $a_{e}$ anomaly in scalar mass $M_{X_{i}}$ versus the magnitude of the $X_{i} e e$ coupling, $\left|g_{i}^{11}\right|$, for scalars (a) $X_{1}$ and (b) $X_{3}$, compared with existing and anticipated experimental constraints. The black dashed line shows our solution for $\Delta a_{e}$ in $\left|g_{X_{i}}^{11}\right|$ with $M_{X_{i}}$ for its experimental central value, with the black band enclosing the solutions bounded by that for $\Delta a_{e}$ taken at $95 \%$ CL. Note that values of $\left|g_{i}^{11}\right|$ above the black band produce a $\left|\Delta a_{e}\right|$ that is too large and are thus excluded by the measurement; we refer to the text for further discussion. We also show the excluded region at $90 \% \mathrm{CL}$ from the measurement of parity-violating Møller scattering from the E158 [87] experiment (solid boundary), as well as the excluded region anticipated from the expected sensitivity of the planned MOLLER experiment (dashed boundary) at Jefferson Laboratory [88,89], if no departure from the SM is observed. We also show the constraint that emerges from measurements of Bhabha scattering at LEP [90] evaluated at 95\% CL-see Sec. V for a detailed discussion.

physics in $p p$ collisions to same-sign dileptons observe no departures from the SM but also require that the dileptoninvariant mass be in excess of $8 \mathrm{GeV}[85,86]$. We note that both $X_{2}$ and $X_{3}$ can induce a contribution to the magnetic moment of a massive Dirac neutrino; we consider this further in Sec. V.

We now summarize our solutions for the $\Delta a_{e}$ anomaly. Working in the $M_{X_{i}} \gg m_{e}$ limit and considering $\Delta a_{e}$ at 95\% confidence level (CL), we find that the masses and scalar-fermion couplings of each $X_{i}$ must satisfy

$$
\begin{aligned}
& 3.2 \times 10^{-6} \leq \frac{m_{e}}{M_{X_{1}}}\left|g_{1}^{11}\right| \leq 9.7 \times 10^{-6}, \\
& 6.5 \times 10^{-6} \leq \frac{m_{e}}{M_{X_{2}}}\left|g_{2}^{1 j}\right| \leq 2.0 \times 10^{-5}, \\
& 3.4 \times 10^{-6} \leq \frac{m_{e}}{M_{X_{3}}}\left|g_{3}^{11}\right| \leq 1.0 \times 10^{-5},
\end{aligned}
$$

where $j \neq 1$. We show the exact numerical solutions for $\left|g_{i}^{11}\right|$ and $M_{X_{i}}$ for $i=1,3$ in Fig. 2, along with other pertinent constraints and their future prospects-the mass range we show is selected to evade both stellar cooling and collider bounds. In this mass range, $X_{2}$, even with the assumption of $1 \leftrightarrow 3$ couplings only, is significantly constrained by branching ratio measurements of semileptonic $\tau$ decay_we update the analysis of Ref. [72] in Sec. V. We develop the established and expected constraints from parity-violating Møller scattering, which act on $X_{1}$ and $X_{3}$, in the next section. Here, we wish to emphasize, in addition to providing the solutions we have shown, that the measured value of $\Delta a_{e}$ also constrains new physics; that is, the upper value of Eqs. (9), (10), and (11) serves as the boundary of a $95 \%$ CL exclusion. That is, we can exclude

$$
\begin{aligned}
\frac{m_{e}}{M_{X_{1}}}\left|g_{1}^{11}\right|>9.7 \times 10^{-6}, \\
\frac{m_{e}}{M_{X_{2}}}\left|g_{2}^{1 j}\right|>2.0 \times 10^{-5}, \\
\frac{m_{e}}{M_{X_{3}}}\left|g_{3}^{11}\right|>1.0 \times 10^{-5},
\end{aligned}
$$

as these regions of parameter space yield values of $\left|\Delta a_{e}\right|$ that are too large - these regions, for $X_{1}$ and $X_{3}$, appear above the shaded black bands in Fig. 2. In contrast, the regions below the black band in Fig. 2 give values of $\left|\Delta a_{e}\right|$ that are too small-although the latter region does not explain the anomaly, these regions of parameter space are not excluded by the $\Delta a_{e}$ result because the scalars we have introduced need not solve the $\Delta a_{e}$ anomaly.

\section{CONSTRAINTS FROM PARITY-VIOLATING MØLLER SCATTERING}

The parity-violating asymmetry $A_{\mathrm{PV}}$ in the low momentum-transfer scattering of longitudinally polarized electrons from unpolarized electrons has been measured to a precision of $17 \mathrm{ppb}$ in the E158 experiment at SLAC, yielding a determination of the value of the effective weak mixing angle $\sin ^{2} \theta_{W}^{\text {eff }}$ to $\simeq 0.5 \%$ precision [87]. In contrast, in a future experiment planned at the Jefferson Laboratory $[88,89]$, the MOLLER Collaboration expects to measure $A_{\mathrm{PV}}$ to an overall precision of $0.7 \mathrm{ppb}$ [89], to determine 
$\sin ^{2} \theta_{W}^{\text {eff }}$ to $\simeq 0.1 \%$ precision [89], with a commensurate improvement of $A_{\mathrm{PV}}$ as a test of new physics. The determination of the weak mixing angle relies on the theoretical assessment of $A_{\mathrm{PV}}$ in the SM [91-96], for which electroweak radiative corrections are important [92-96]. Nevertheless, as per usual practice [74,88,89], we use the tree-level formula for $A_{\mathrm{PV}}$ of Ref. [91] to determine the sensitivity of the existing and planned $A_{\mathrm{PV}}$ measurements to new physics. Only the doubly charged scalars, $X_{1}$ and $X_{3}^{3}$, couple to two electrons, so that they contribute in the $s$ channel to Møller scattering, i.e., via $e^{-}(p)+e^{-}(k) \rightarrow X_{i} \rightarrow e^{-}\left(p^{\prime}\right)+e^{-}\left(k^{\prime}\right)$. Since we are considering constraints on light scalars, the value of $s$ is important: we note that both E158 and MOLLER are fixedtarget experiments with an electron beam energy of $E=$ $50 \mathrm{GeV}$ for E158 [87] and $E=12 \mathrm{GeV}$ for the MOLLER experiment [89]. Thus, we have $s \simeq 2 m_{e} E$, with $\sqrt{s} \simeq$ $0.23 \mathrm{GeV}$ for E158-we label this " $\sqrt{s^{\prime}}$ " in Fig. 2-and $\sqrt{s} \simeq 0.11 \mathrm{GeV}$ for MOLLER. If a measured value of $A_{\mathrm{PV}}$ agrees with SM expectations, then a modelindependent constraint on new four-electron contact interactions follows, such as those of either left-left or right-right form [97]

$$
\mathcal{H}_{\text {new }}=-\frac{g_{\xi \xi}^{2}}{2 \Lambda^{2}} \overline{\psi_{\xi}} \gamma^{\mu} \psi_{\xi} \overline{\psi_{\xi}} \gamma_{\mu} \psi_{\xi}
$$

for $\xi=L, R$. For the MOLLER experiment, e.g., we would have the lower bound $[88,89]$

$$
\frac{\Lambda}{\sqrt{\left|g_{R R}^{2}-g_{L L}^{2}\right|}}=\frac{1}{\sqrt{\sqrt{2} G_{F}\left|\Delta Q_{W}^{e}\right|}} \simeq 7.5 \mathrm{TeV},
$$

at $67 \%$ C.L., where $\Lambda$ is the mass scale of new physics and $G_{F}$ is the Fermi constant. We note that the error in the weak charge of the electron $\Delta Q_{W}^{e}$, where $Q_{W}^{e} \equiv 1-4 \sin ^{2} \theta_{W}^{\text {eff }}$ [89] in the $\mathrm{SM}$, is $\pm 5.1 \times 10^{-3}$ for the E158 experiment [87] and is expected to be $\pm 1.1 \times 10^{-3}$ for the MOLLER experiment [89]. Interpreting both results at 90\% C.L. yields $\Lambda / \sqrt{\left|g_{R R}^{2}-g_{L L}^{2}\right|} \simeq 2.7 \mathrm{TeV}$ and $\Lambda / \sqrt{\left|g_{R R}^{2}-g_{L L}^{2}\right|} \simeq$ $5.7 \mathrm{TeV}$ for the E158 and MOLLER experiments, respectively.

Returning to the possibility of doubly charged scalars, we rewrite the interactions of Eq. (5) as

$$
\mathcal{H} \supset g_{i}^{11} X_{i} \overline{\psi^{c}} P_{\xi_{i}} \psi+g_{i}^{11 *} X_{i}^{*} \bar{\psi} P_{-\xi_{i}} \psi^{c},
$$

where $i$ denotes model 1 or 3 . Here, $\xi_{1},-\xi_{1}$ are $R, L$, and $\xi_{3},-\xi_{3}$ are $L, R$. Computing the $S$ matrix for Møller scattering, $e^{-}(p)+e^{-}(k) \rightarrow e^{-}\left(p^{\prime}\right)+e^{-}\left(k^{\prime}\right)$,

$$
\left\langle\boldsymbol{p}^{\prime} \boldsymbol{k}^{\prime}\left|T\left(\frac{1}{2 !}(-i)^{2} \int d^{4} x \mathcal{H}(x) \int d^{4} y \mathcal{H}(y)\right)\right| \boldsymbol{p} \boldsymbol{k}\right\rangle,
$$

and noting that $\overline{\psi^{c}}(x) P_{\xi} \psi(x) \bar{\psi}(y) P_{\xi^{\prime}} \psi^{c}(y)$ and $\overline{\psi^{c}}(y) \times$ $P_{\xi} \psi(y) \bar{\psi}(x) P_{\xi^{\prime}} \psi^{c}(x)$ generate the same contribution to the $S$ matrix, we have

$$
\begin{gathered}
-\left|g_{i}^{11}\right|^{2}\left\langle\boldsymbol{p}^{\prime} \boldsymbol{k}^{\prime}\right| T\left(\int d^{4} x X_{i}(x) \overline{\psi^{c}}(x) P_{\xi} \psi(x)\right. \\
\left.\times \int d^{4} y X_{i}^{*}(y) \bar{\psi}(y) P_{\xi^{\prime}} \psi^{c}(y)\right)|\boldsymbol{p} \boldsymbol{k}\rangle .
\end{gathered}
$$

After contracting $X_{i}$ and $X_{i}^{*}$, applying a Fierz transformation [98], and working in the $s \ll M_{X_{i}}^{2}$ limit, we extract the effective Hamiltonian

$$
\mathcal{H}_{\mathrm{eff}}=\frac{-\left|g_{i}^{11}\right|^{2}}{2 M_{i}^{2}} \bar{\psi} \gamma^{\mu} P_{\xi} \psi \bar{\psi} \gamma_{\mu} P_{\xi} \psi
$$

Comparing with Eq. (15), we identify $g_{R R} \equiv\left|g_{1}^{11}\right|$ and $g_{L L} \equiv\left|g_{3}^{11}\right|$. For definiteness, we note that Eq. (15) follows from the use of the $Z_{0}$ interaction in Ref. [91] to compute $A_{\mathrm{PV}}$, with $v=g_{R R}+g_{L L}, a=g_{R R}-g_{L L}, g_{0}=1 / 2$, which also yields $\left|g_{R R}^{2}-g_{L L}^{2}\right| / \Lambda^{2} \leftrightarrow \sqrt{2} G_{F}\left|\Delta Q_{W}^{e}\right|$ as used in Eq. (16). Previously, the relations $\left|g_{R R}\right|^{2} \equiv\left|g_{1}^{11}\right|^{2} / 2$ and $\left|g_{L L}\right|^{2} \equiv\left|g_{3}^{11}\right|^{2} / 2$ were used to set the effective mass scale $\Lambda$ for the doubly charged scalars [74,88,89]; however, as we have shown, those $2 \mathrm{~s}$ should not appear. In our current analysis, we wish to constrain light scalars, so that $s \ll M_{X_{i}}^{2}$ no longer needs to be satisfied. We note that we may still safely use $A_{\mathrm{PV}}$ as computed in Ref. [91] because $g_{\xi \xi}^{2} s /\left(2\left(s-M_{X_{i}}^{2}\right) \pi \alpha\right) \ll 1$ can be satisfied nonetheless. Thus, at low scales, we replace $\Lambda / \sqrt{\left|g_{R R}^{2}-g_{L L}^{2}\right|}$ by $\sqrt{\left|s-M_{X_{i}}^{2}\right|} /\left|g_{i}^{11}\right|$ to find the constraints

$$
\frac{\sqrt{\left|s-M_{X_{i}}^{2}\right|}}{\left|g_{i}^{11}\right|} \gtrsim 2.7 \mathrm{TeV}, \quad \frac{\sqrt{\left|s-M_{X_{i}}^{2}\right|}}{\left|g_{i}^{11}\right|} \gtrsim 5.7 \mathrm{TeV},
$$

at $90 \%$ C.L. for the E158 [87] and MOLLER experiments [89], respectively. Thus, we note that the constrained region depends on the c.m. energy for each experiment and that if $M_{X_{i}} \ll \sqrt{s}$ only the coupling constants $g_{i}^{11}$ are constrained. In particular, if $M_{X_{i}} \ll \sqrt{s^{\prime}}$, the E158 constraint becomes $\left|g_{i}^{11}\right| \leq 8.58 \times 10^{-5}$, whereas if $M_{X_{i}} \ll \sqrt{s}$, the MOLLER constraint becomes $\left|g_{i}^{11}\right| \leq 1.9 \times 10^{-5}$. The exclusion limits from Eq. (21) as a function of $M_{X_{i}}$ are shown in Fig. 2. One can find that indeed both the solid (red) and dashed (olive) curves become straight lines as $M_{X_{i}}$ grows much bigger than $\sqrt{s^{\prime}}$ and $\sqrt{s}$. Moreover, as $M_{X_{i}}$ becomes much smaller than $\sqrt{s^{\prime}}$ and $\sqrt{s}$, the solid (blue) and dashed (purple) curves become flat, so that only a coupling constant constraint emerges. (Note that the constraint from 
precision measurements of Bhabha scattering at LEP [90] is also a coupling constant constraint because the c.m. energies studied far exceed the scalar masses of interest [99]; we refer to Sec. V for a detailed discussion.) In the regions for which $M_{X_{i}}$ is very close to either $\sqrt{s}$ or $\sqrt{s^{\prime}}$, the constraints of Eq. (21) demand a very small coupling constant, though the evaluation of $A_{\mathrm{PV}}$ can become nontrivial - it may be necessary to replace the scalar propagator by a Breit-Wigner form to find a definite result. However, for the region shown in Fig. 2, this is not needed.

\section{OTHER CONSTRAINTS}

Light scalars that carry lepton number and couple to electrons, in a manner that preserves SM symmetries, also carry electric charge. As a result, the "beam-dump" experiments that severely constrain the electron coupling to electrically neutral, light scalars $[41,100]$ do not operate because electrically charged scalars interact with the material of the target or beam dump and do not escape. Certainly, too, searches for $s$-channel resonances in lowenergy Bhabha scattering [101] do not apply to the current case (and we consider the impact of new scalars in Bhabha scattering in the $t$ channel later in this section), though an analogous search for a low-energy, $s$-channel resonance in $e^{-} e^{-}$scattering should be possible, though the extremely narrow decay widths associated with the scalar solutions we have found in Fig. 2 may make a sufficiently sensitive test impracticable. In what follows, we consider further constraints particular to scalars that carry lepton number.

The scalars $X_{1}$ and $X_{2}$ have been previously discussed in the context of a particular model $[67,68]$ in which the neutrino masses are generated through radiative corrections $[64,66]$. In this paper, we do not delve into the origin of neutrino masses. Nevertheless, the scalars $X_{2}$ and $X_{3}^{2}$ can potentially mediate additional neutrino mass contributions. We find it impossible to generate either a Dirac or Majorana neutrino mass at one-loop level, so that our flavor-specific couplings do not in themselves impact the neutrino mass splittings. However, if both $X_{1}$ and $X_{2}$ exist, then a minimal scalar interaction of form $\mu X_{2} X_{2} X_{1}^{*}+$ H.c. can also exist between them, ${ }^{2}$ and then it is possible to induce a neutrino Majorana mass at two-loop order $[67,68,72]$. The mass prediction depends on the size of $\mu$, the coupling constant associated with the scalar-scalar interaction, and although its upper bound has been estimated in Ref. [72], there are no constraints on its minimum value-thus these considerations do not restrict the parameter space of interest to us in this paper.

If neutrinos are massive Dirac particles, then the scalars $X_{2}$ and $X_{3}^{2}$ can each contribute to its magnetic moment, though these effects turn out to be extremely small. The

\footnotetext{
${ }^{2}$ This interaction is the same as model $\mathrm{F}$ in our recent work [51].
}

largest contributions in the region of parameter space of interest to us come from $X_{2}$ to $\mu_{\nu_{\tau}}$ if $M_{X_{2}} \simeq m_{e}$ and from $X_{3}^{2}$ to $\nu_{e}$ if $M_{X_{3}} \simeq m_{e}$. Employing Eq. (A35), we find

$\left[\delta \mu_{\nu_{\tau}}\right]_{X_{2}} \simeq \frac{-1}{12} \frac{\left|g_{2}^{13}\right|^{2}}{\pi^{2}} \frac{m_{\nu}^{2}}{m_{e}^{2}} \mu_{B}, \quad\left[\delta \mu_{\nu_{e}}\right]_{X_{3}} \simeq \frac{-1}{24} \frac{\left|g_{3}^{11}\right|^{2}}{\pi^{2}} \frac{m_{\nu}^{2}}{m_{e}^{2}} \mu_{B}$

where for simplicity we have assumed the neutrinos are approximately degenerate, with mass $m_{\nu}$ and $\mu_{B}$ the Bohr magneton. From cosmological observations, we have $\sum_{j} m_{j}<0.170 \mathrm{eV}$ at $95 \%$ C.L., though the best current limit on $m_{\bar{\nu}_{e}}$ from ${ }^{3} \mathrm{H} \beta$-decay is $m_{\bar{\nu}_{e}}<2.05 \mathrm{eV}$ at $95 \%$ C.L. [7]. Thus, we see that, even with $m_{\nu} \simeq 2 \mathrm{eV}$ and $\left|g_{2}^{13}\right|=1$, the largest contribution, $\left[\delta \mu_{\nu_{\tau}}\right]_{X_{2}}$, cannot be excluded by the current best experimental limit $|\mu|_{\nu} \sim 2.9 \times 10^{-11} \mu_{B}$ [102] nor by expected improvements $[103,104]$.

We now turn to the consideration of constraints from flavor physics, noting the comprehensive analysis of Ref. [72]. Taken altogether, the constraints on flavornondiagonal scalar-fermion couplings from the experimental limits on lepton flavor-violating processes, and from the muon lifetime, are severe. As a result, we have considered first-generation couplings for $X_{1}$ and $X_{3}$, and first-third generation couplings for $X_{2}$ exclusively. Consequently, we need only consider the constraint from the measurement of $\tau \rightarrow e \bar{\nu}_{e} \nu_{\tau}$ decay, as the only other constraint, from $e / \mu$ lepton-flavor universality in semileptonic $\tau$ decay, acts similarly.

The scalar $X_{2}$ can mediate $\tau$ semileptonic decay via $\tau(p) \rightarrow \bar{\nu}_{e} X_{2}^{*} \rightarrow \bar{\nu}_{e}\left(k^{\prime}\right) e^{-}\left(p^{\prime}\right) \nu_{\tau}(k)$. After a Fierz transformation, we find the decay amplitude can found from the SM result by replacing $G_{F}^{2} \rightarrow\left|g_{2}^{13}\right|^{4} /\left[2\left(t-M_{X_{2}}^{2}\right)^{2}\right]$, where $t=\left(p-k^{\prime}\right)^{2}$. Working in the $\tau$ rest frame and integrating over the three-body phase space, neglecting all the light lepton masses, yields

$$
\Gamma=\frac{m_{\tau}\left|g_{2}^{13}\right|^{4}}{4 \pi^{3}} \int_{0}^{m_{\tau} / 2} d \omega^{\prime} \frac{\left(\omega^{\prime}\right)^{2}\left(m_{\tau}-2 \omega^{\prime}\right)}{\left(m_{\tau}^{2}-2 m_{\tau} \omega^{\prime}-M_{X_{2}}^{2}\right)^{2}},
$$

where $\omega^{\prime}$ is the energy of the antielectron neutrino. For $M_{X_{2}}>m_{\tau}$, the integral is well defined and for $M_{X_{2}} \gg m_{\tau}$ yields the familiar result

$$
\Gamma=\frac{m_{\tau}^{5}}{192 \pi^{3}} \frac{\left|g_{2}^{13}\right|^{4}}{2 M_{X_{2}}^{4}} .
$$

For $M_{X_{2}}<m_{\tau}$, a $t$-channel pole appears, which we address by replacing the scalar propagator by a Breit-Wigner form:

$$
\frac{1}{\left(t-M_{X_{2}}\right)^{2}} \rightarrow \frac{1}{\left|t-M_{X_{2}}^{2}+i M_{X_{2}} \Gamma_{X_{2}}\right|^{2}} .
$$


Defining

$$
x=\frac{t}{m_{\tau}^{2}}, \quad x_{X}=\frac{M_{X_{2}}^{2}}{m_{\tau}^{2}}, \quad \tilde{\Gamma}_{X}=\frac{\Gamma_{X_{2}}}{m_{\tau}},
$$

we thus have

$$
\Gamma=\frac{m_{\tau}\left|g_{2}^{13}\right|^{4}}{32 \pi^{3}} \int_{0}^{1} d x \frac{(1-x)^{2} x}{\left(x-x_{X}\right)^{2}+x_{X} \tilde{\Gamma}_{X}^{2}} .
$$

Since $x_{X} \tilde{\Gamma}_{X}^{2} \ll 1$, we can apply the narrow width approximation [105], i.e.,

$$
\left(\left(x-x_{A^{\prime}}\right)^{2}+x_{A^{\prime}} \tilde{\Gamma}_{A^{\prime}}^{2}\right)^{-1} \rightarrow \frac{\pi}{\sqrt{x_{A^{\prime}} \tilde{\Gamma}_{A^{\prime}}}} \delta\left(x-x_{A^{\prime}}\right),
$$

to find

$$
\Gamma=\frac{m_{\tau}\left|g_{2}^{13}\right|^{4}}{32 \pi^{2}} \frac{m_{\tau} M_{X_{2}}}{\Gamma_{X_{2}}}\left(1-\frac{M_{X_{2}}^{2}}{m_{\tau}^{2}}\right)^{2} .
$$

Since there is only one decay channel left for $X_{2}$, $X_{2}^{*} \rightarrow e^{-} \nu_{\tau}$, we compute

$$
\Gamma_{X_{2}}=\frac{1}{4 \pi} M_{X_{2}}\left|g_{2}^{13}\right|^{2}
$$

to find

$$
\Gamma=\frac{m_{\tau}\left|g_{2}^{13}\right|^{2}}{8 \pi}\left(1-\frac{M_{X_{2}}^{2}}{m_{\tau}^{2}}\right)^{2},
$$

which, as expected, is identical to our result for $\Gamma\left(\tau \rightarrow e X_{2}^{*}\right)$. We now turn to the numerical constraints on the scalar-fermion couplings with $M_{X_{2}}$, given existing measurements of the $\tau \rightarrow e \bar{\nu}_{e} \nu_{\tau}$ branching ratio and $\tau$ lifetime. Referring to Ref. [7] for all experimental parameters, we note particularly that $\operatorname{Br}\left(\tau \rightarrow e \bar{\nu}_{e} \nu_{\tau}\right)=$ $17.82 \pm 0.04 \%, \tau_{\tau}=(290.3 \pm 0.5) \times 10^{-15} \mathrm{~s}$, and $m_{\tau}=$ $1776.86 \pm 0.12 \mathrm{MeV}$. For $M_{X} \gg m_{\tau}$, we can constrain, at $90 \%$ C.L.,

$$
\frac{\left|g_{2}^{13}\right|^{4}}{2 M_{X_{2}}^{4} G_{F}^{2}} \leq \frac{\eta}{\operatorname{Br}\left(\tau \rightarrow e \bar{\nu}_{e} \nu_{\tau}\right)} \Rightarrow \frac{\left|g_{2}^{13}\right|}{M_{X_{2}}} \leq 1.0 \times 10^{-3} \mathrm{GeV}^{-1}
$$

or

$$
\frac{m_{\tau}^{5}}{192 \pi^{3}} \frac{\left|g_{2}^{13}\right|^{4}}{2 M_{X_{2}}^{4}} \leq \frac{\eta h}{100 \tau_{\tau}} \Rightarrow \frac{\left|g_{2}^{13}\right|}{M_{X_{2}}} \leq 1.6 \times 10^{-3} \mathrm{GeV}^{-1}
$$

with $\eta=0.066$. The two estimates differ in that the former implicitly assumes the leading-order formula describes the SM decay rate, though various refinements exist [106].

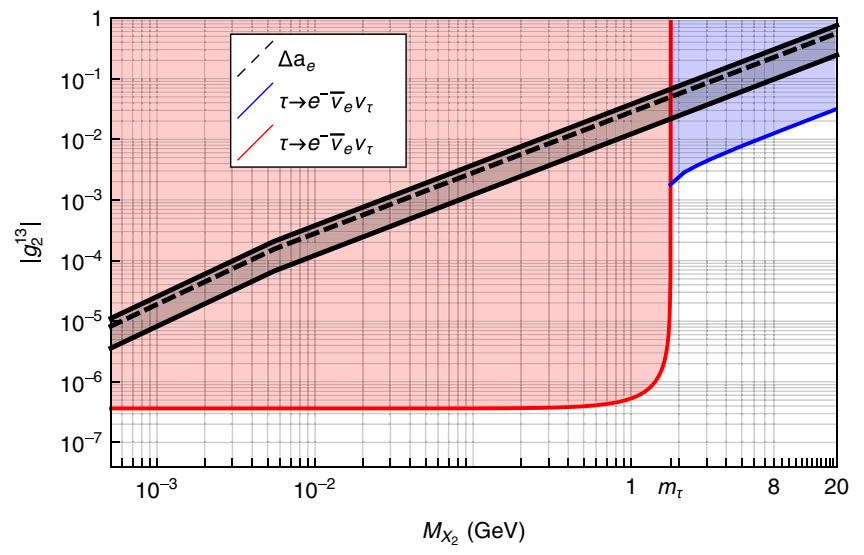

FIG. 3. Our solution for the $a_{e}$ anomaly in scalar mass $M_{X_{2}}$ versus the magnitude of the $X_{2} e \nu_{\tau}$ coupling, $\left|g_{2}^{13}\right|$, compared with existing experimental constraints. In this case, we have shown our solution over a larger mass range than in Fig. 2 because the collider constraints on same-sign dileptons do not apply $[85,86]$. The black dashed line and band are defined as in Fig. 2 but are for $\Delta a_{e}$ in $\left|g_{2}^{13}\right|$ with $M_{X_{2}}$. We also show the experimentally excluded region at $90 \%$ C.L. from the current error in the measured branching ratio in $\tau \rightarrow e \bar{\nu}_{e} \nu_{\tau}$ decay [7]; for $M_{X_{2}}<m_{\tau}$, we assume that the $X_{2}$ width is saturated by $X_{2} \rightarrow e^{-} \bar{\nu}_{\tau}$ decay and refer to the text for further discussion.

We note that the numerical limit reported by Ref. [72] in this case is significantly more severe than what we report. In what follows, we use our second method to determine the exclusion limit. For $M_{X_{2}}>m_{\tau}$, we replace the lhs of Eq. (33) with Eq. (23). For $M_{X_{2}}<m_{\tau}$, we replace the lhs of Eq. (33) with Eq. (31). We report the $90 \%$ C.L. exclusion we have found in Fig. 3, recalling that $\left(\delta a_{e}\right)_{X_{2}}<0$ only if $M_{X_{2}}>m_{e}$. Thus, we see that in this case the existing empirical data rule out $X_{2}$ as a solution to the $a_{e}$ anomaly, at least in a minimal scalar model. More generally, we note that Eq. (31) can be written as [105]

$$
\Gamma=\frac{m_{\tau}\left|g_{2}^{13}\right|^{4}}{8 \pi}\left(1-\frac{M_{X_{2}}^{2}}{m_{\tau}^{2}}\right)^{2} \operatorname{Br}\left(X_{2}^{*} \rightarrow e^{-} \nu_{\tau}\right)
$$

and that decreasing $\operatorname{Br}\left(X_{2}^{*} \rightarrow e^{-} \nu_{\tau}\right)$ from unity weakens the constraint on $\left|g_{2}^{13}\right| / M_{X_{2}}$ in the $M_{X_{2}}<m_{\tau}$ region.

Finally, we turn to the constraints that appear because our scalars couple to the gauge bosons of the SM. The doubly charged scalars that we consider are constrained just as the doubly charged Higgs bosons $H_{L, R}^{ \pm \pm}[65,107-109]$ in generalized left-right symmetric models [110] are. In what follows, the constraints on $H_{R}^{ \pm \pm}\left(H_{L}^{ \pm \pm}\right)$are identical to those on $X_{1}\left(X_{3}^{3}\right)$. We note that the same-sign dilepton limits from searches for $p p[q \bar{q}] \rightarrow H_{L, R}^{ \pm \pm} H_{L, R}^{\mp \mp} \rightarrow \ell^{ \pm} \ell^{ \pm} \ell^{\mp} \ell^{\mp}$ from the LHC at $\sqrt{s}=13 \mathrm{TeV}$ with $\ell \in e, \mu$ yield $M_{H_{L}^{++}}>768 \mathrm{GeV}$ at $95 \%$ C.L. and $M_{H_{R}^{++}}>658 \mathrm{GeV}$ at 95\% C.L. for $\operatorname{Br}\left(H_{L, R}^{++} \rightarrow e^{+} e^{+}\right)=1[111,112]$, where the 
experiments are most sensitive to doubly charged scalars with masses in excess of $200 \mathrm{GeV}$ - e.g., the same-sign dilepton invariant mass is required to be in excess of $200 \mathrm{GeV}$ in the study of the $e^{ \pm} e^{ \pm} e^{\mp} e^{\mp}$ final state [111]. Thus, to constrain lighter mass scalars, we must look further. Extensive searches for charged scalars have been made at LEP [113]. Such measurements can probe doubly charged scalars over a very wide mass range, both indirectly, through $t$-channel exchange of $H^{ \pm \pm}$in Bhabha scattering $[90,99,114]$, and directly, through associated production, $e^{+} e^{-} \rightarrow e^{ \pm} e^{ \pm} H^{\mp \mp}$ [114]. The latter process tends to be more sensitive to the size of the Higgs coupling to electrons $h_{e e}$ (our $g_{1}^{11}$ or $g_{3}^{11}$ ), but the former is sensitive to a much broader range of masses. In these experiments, no evidence for the existence of $H^{ \pm \pm}$has been found, with an upper limit of $h_{e e}<0.071$ at 95\% C.L. inferred for $M_{H^{ \pm \pm}}<160 \mathrm{GeV}$ coming from their direct search, though the region with $M_{H^{ \pm \pm}}<98.5 \mathrm{GeV}$ had been assumed to be excluded by studies of pair production. In particular, the direct search did not search for doubly charged scalars less than $80 \mathrm{GeV}$ in mass [114]. Turning to the pair production studies, through $e^{+} e^{-}$scattering in the $s$ channel [115], a mass limit of $98.5 \mathrm{GeV}$ at $95 \%$ C.L. has indeed been set, but a lower mass limit of $45 \mathrm{GeV}$ is assumed from $Z^{0}$ decay studies [115]. We note that doubly charged scalars have been studied in $Z^{0}$ decay, $Z^{0} \rightarrow H^{++} H^{--}$[116]. The experiment is unable to constrain scalars with masses of less than a few $\mathrm{GeV}$ directly, and constraints on the mass of $H^{ \pm \pm}$are found by appealing to measurements of the $Z^{0}$ line shape. That is, they determine that the difference between the $Z^{0}$ width measurement and its SM prediction is less than $40 \mathrm{MeV}$ at $95 \%$ C.L., so that a bound on the doubly charged scalar mass is set by requiring that the $Z^{0} \rightarrow$ $\mathrm{H}^{++} \mathrm{H}^{--}$partial width is no larger than $40 \mathrm{MeV}$ [116]. In this way, they finally determine the mass exclusion limits of less than $25.5 \mathrm{GeV}$ for weak-isospin singlets (our $X_{1}$ ) and of less than $30.4 \mathrm{GeV}$ for weak-isospin triplets (our $X_{3}$ ) at 95\% C.L. [116] using [110]

$$
\begin{aligned}
\Gamma\left(Z^{0} \rightarrow H^{++} H^{--}\right)= & \frac{G_{F} M_{Z}^{3}}{6 \pi \sqrt{2}}\left(I_{3}^{L}-Q \sin ^{2} \theta_{W}\right)^{2} \\
& \times\left(1-\frac{4 M_{H}^{2}}{M_{Z}^{2}}\right)^{3 / 2},
\end{aligned}
$$

where $M_{H}, Q$, and $I_{3}^{L}$ are the mass, charge, and the third component of weak isospin of the $H^{ \pm \pm}$. For the righthanded singlet, we set $I_{3}^{L}=0$. We can easily mitigate this constraint, however, through an addition to our model, as we detail below. There is also a pair production constraint extracted from $e^{+} e^{-} \rightarrow e^{+} e^{-} \ell^{+} \ell^{-}$data measured by the CELLO Collaboration at PETRA to realize tests of QED [117], which Swartz has analyzed to determine a limit of $21.5 \mathrm{GeV}$ at $90 \%$ C.L. on the mass of the doubly charged scalar if $\operatorname{Br}\left(H^{ \pm \pm} \rightarrow e^{ \pm} e^{ \pm}\right)=1$ [99]. The decay width of the doubly charged scalar is given by [99]

$$
\Gamma_{\ell \ell}=\frac{h_{\ell \ell}^{2}}{8 \pi} M_{H}\left(1-2 \frac{m_{\ell}^{2}}{M_{H}^{2}}\right)\left(1-4 \frac{m_{\ell}^{2}}{M_{H}^{2}}\right)^{1 / 2},
$$

and Ref. [99] notes that the doubly charged scalar can be short lived unless $h_{\ell \ell}<10^{-9}$. However, this observation does not bear out for lighter mass scalars. In the empirical study of $e^{+} e^{-} \rightarrow e^{+} e^{-} \ell^{+} \ell^{-}$by Le Diberder [117], three out of the four final state leptons were detected under the requirement of a "good vertex" (as per Eq. (A-1.3) of Ref. [117]) in order to control backgrounds. As a result, a produced doubly charged scalar with a decay length in excess of $0.4 \mathrm{~cm}$ would not have been detected by the experiment. We find that this requirement removes light, weakly coupled scalars from the aforementioned constraint. Namely, requiring that the decay length in the laboratory frame satisfies

$$
\left(\frac{\sqrt{s}}{2 M_{H}} \sqrt{1-\frac{4 M_{H}^{2}}{s}}\right) \frac{\hbar c}{\Gamma_{\ell \ell}}<0.4 \mathrm{~cm},
$$

we see that for $\sqrt{s}$ of $40 \mathrm{GeV}$, e.g., if $M_{H}=1 \mathrm{GeV}$, then couplings with $h_{e e}>5.0 \times 10^{-6}$ are excluded, whereas if $M_{H}=100 \mathrm{MeV}(10 \mathrm{MeV})$, then the exclusion limit changes to $h_{e e}>5.0 \times 10^{-5}\left(5.0 \times 10^{-4}\right)$. Thus, we observe that our possible $(g-2)_{e}$ solutions are not constrained by the PETRA data. From our discussion, we observe that the only significant constraint on the mass of the light scalar comes from the measured width of the $Z^{0}$ gauge boson.

Further constraints come from the indirect process, Bhabha scattering. In this case, if $M_{H}^{2} \ll s$ [99], the indirect process becomes insensitive to the mass of the scalar, much like we have seen in the case of Møller scattering, constraining only the $h_{e e}$ coupling constant in this limit. We note the limit of $h_{e e}<0.14$ at 95\% C.L. from $e^{+} e^{-} \rightarrow$ $e^{+} e^{-}$collision data at c.m. energies of $\sqrt{s}=183-209 \mathrm{GeV}$ collected by the OPAL detector [114]. Moreover, $e^{+} e^{-} \rightarrow$ $e^{+} e^{-}$cross section measurements at c.m. energies of $\sqrt{s} \sim$ 130-207 GeV at LEP by the DELPHI Collaboration yield a limit of $h_{e e}<0.088$ at 95\% C.L., determined from their limit on a new contact interaction of the form in Eq. (20), with $M_{i} \equiv \Lambda^{-}=6.8 \mathrm{TeV}$ for LL and RR from Table 30 for a coupling of strength $g=\sqrt{4 \pi}$ [90]. This last limit is reported in Fig. 2.

Finally, since our scalars can carry electric charge, we evaluate the indirect constraints on them that follow from the direct measurement of the running of $\alpha(s)$, $|\alpha(s) / \alpha(0)|^{2}$, where $\alpha \equiv \alpha(0)$. This can be determined

${ }^{3}$ The experiment employed beam energies from 17.5 to $23 \mathrm{GeV}$ [99]. 
from the measured differential cross section for $e^{+} e^{-} \rightarrow$ $\mu^{+} \mu^{-} \gamma$, for which the most precise results are in the timelike region below $1 \mathrm{GeV}$ [118] — there, the presence of hadronic contributions is established at more than $5 \sigma$. We evaluate $\alpha(s)=\alpha /(1-\Delta \alpha(s))$ [119], where the leading contribution to the vacuum polarizaton $\Delta \alpha$ can be readily calculated in scalar QED to yield [9]

$$
\begin{aligned}
\operatorname{Re} \Delta \alpha_{X_{2}}(s) & =-\frac{\alpha}{2 \pi} \int_{0}^{1} d x x(2 x-1) \log \left|\frac{M_{X_{2}}^{2}}{M_{X_{2}}^{2}-s x(1-x)}\right|, \\
& =\frac{\alpha}{12 \pi}\left[\log \left(\frac{s}{M_{X_{2}}^{2}}\right)-\frac{8}{3}\right] \quad \text { for } s \gg 4 M_{X_{2}}^{2} ;
\end{aligned}
$$

$\operatorname{Im} \Delta \alpha_{X_{2}}(s)=-i \frac{\alpha}{12}\left(1-\frac{4 M_{X_{2}}^{2}}{s}\right)^{3 / 2} \Theta\left(s-4 M_{X_{2}}^{2}\right)$.

We note that $\Delta \alpha_{X_{2}}(s)$ is four times smaller, and $\operatorname{Re} \Delta \alpha_{X_{2}}(s)$ runs more slowly, than that for a fermion in QED. The contribution of $X_{2}$ for $M_{X_{2}} \leq m_{e}$ to $|\alpha(s) / \alpha(0)|^{2}$ deviates from unity by less than $0.5 \%$ over the $s$ range of the experiment, $0.6<\sqrt{s}<0.975 \mathrm{GeV}$, with an inappreciable $s$ dependence. Since the individual measurements have a statistical error of less than or equal to $1 \%$ and an overall systematic error of $1 \%$ [118], the existence of the $X_{2}$ scalar is not constrained. However, the contributions from $X_{1}$ and $X_{3}$ include doubly charged scalars, and we have $\Delta \alpha_{X_{1}}(s)=$ $4 \Delta \alpha_{X_{2}}(s)$ and $\Delta \alpha_{X_{3}}(s)=5 \Delta \alpha_{X_{2}}(s)$. Although the contributions to $\alpha(s)$ from $X_{1}$ and $X_{3}$ also have negligibly small slope in the $s$ range of interest, they can each generate an appreciable offset from zero. We suppose that the existence of these scalars is limited by the size of the overall systematic error, or offset, in the measurement of $|\alpha(s) / \alpha(0)|^{2}$. Noting the measured data points and their errors in Table 2 of Ref. [118], we require that the overall shift in the theory contribution with a new scalar is less than 0.011 for $\sqrt{s}<0.783 \mathrm{GeV}$, the region for which the hadronic contribution is completely captured by the included $2 \pi$ intermediate state. Thus, we estimate $M_{X_{1}}>$ $8.4 \mathrm{MeV}$ and $M_{X_{3}}>19 \mathrm{MeV}$. We regard these limits as guidelines rather than exclusions because the new scalars generate contributions that do not impact the measured $s$ dependence but, rather, only its overall normalization. Nevertheless, this analysis suggests that $X_{1}$ is a more likely solution to the $(g-2)_{e}$ anomaly.

We have found severe constraints on the allowed doubly charged scalar mass from its couplings to the $Z^{0}$ and to the photon, notably through the running of $\alpha$. We note that the $Z^{0}$ constraint, in particular, can be readily mitigated through the introduction of a higher-dimension operator that acts to neutralize the couplings of the doubly charged scalars to SM gauge bosons. That is, we can add an operator of the form

$$
-g_{\Phi} \frac{|\Phi|^{2}\left|D_{\mu} X_{i}\right|^{2}}{\Lambda_{\Phi}^{2}}
$$

where the scalar $\Phi$ is an electroweak singlet with zero $\mathrm{L}$ and zero electric charge. We let $\Phi$ gain a vacuum expectation value $v_{\Phi}$ below the scale $\Lambda_{\Phi}$, where $v_{\Phi} \sim \Lambda_{\Phi}$ exceeds the electroweak scale and the coefficient $-g_{\Phi} v_{\Phi}^{2} / \Lambda_{\Phi}^{2}$, with $g_{\Phi}>0$, acts to neutralize the lepton number-carrying scalars' $\mathrm{SU}(2)_{L}$ and electric charges. Turning to Eq. (35) and considering the limit on $X_{1}$, under which, e.g., the factor $\left(1-4 M_{H}^{2} / M_{Z}^{2}\right)^{3 / 2}$ evaluates to 0.58 , we see that by weakening the effective $\mathrm{SU}(2)$ coupling of the $X_{1}$ by about $20 \%$ we would be able to remove this constraint completely. This seems plausibly attainable, and we note that such a change makes only a trivially small impact on the $\Delta a_{e}$ solutions we show in Fig. 2 because the contributions of the charged scalars themselves to $a_{e}$ are numerically quite small. Thus, we have not included this effect in Fig. 2.

\section{SUMMARY}

In this paper, we have shown that the light scalars with lepton number that appear in minimal scalar models of new physics can generate solutions to the $\Delta a_{e}$ anomaly, in that they act to reduce the size of $\left|a_{e}\right|$. Although our solutions determine only the ratio of the scalar-fermion coupling to mass, we have particularly focussed on new particles with masses in excess of the electron mass and less than $8 \mathrm{GeV}$, as this mass region, at first glance, should evade both astrophysical cooling constraints and collider bounds. We should note, however, that, since the scalars that couple to electrons also carry electric charge, lighter mass candidates could also prove phenomenologically viable, because such particles may be unable to escape an astrophysical environment and contribute to its cooling. We have proposed three possible solutions to the $\Delta a_{e}$ anomaly, but we have found that only the two solutions with doubly charged scalars are viable because the existing $\tau$ decay data preclude the singly charged scalar $X_{2}$ as a possible solution, at least in a minimal scalar model. As for the doubly charged scalars, the constraints from parity-violating Møller scattering permit a solution to the $\Delta a_{e}$ anomaly, with the upcoming MOLLER experiment poised to discover a conflict with the SM or to constrain our proposed solutions yet further. We have also carefully studied existing collider constraints on doubly charged scalars, and we have noted that the only pertinent constraint on the solutions we consider comes from studies of the $Z^{0}$ line width. We can readily weaken this constraint as needed through the addition of a higher-dimension operator that acts to neutralize the $\mathrm{SU}(2)$ and electric charges of the doubly charged scalar boson, and this addition leaves the parameters of our proposed $\Delta a_{e}$ solutions essentially unchanged.

We have noted, moreover, that the $\Delta a_{e}$ determination also constrains broad swatches of the scalar-fermion 
coupling and mass parameter space, as parameters which would give too large a value of $\left|a_{e}\right|$ should be excluded. There are plans to make substantially improved measurements of both the electron and the positron anomalous magnetic moments [17], to better existing measurements by a factor of 10 and 150 [120], respectively. Although this comparison is meant as a $C P T$ test, it can also help affirm our new physics solution to the $\Delta a_{e}$ anomaly, as the two new measurements could well agree with each other, up to the expected difference in overall sign, but yet disagree with the SM using $\alpha$ determined through atom interferometry. The scalar solutions we have found can also help engender baryon- and lepton-number violation in lowenergy scattering experiments, and we keenly await these studies.

\section{ACKNOWLEDGMENTS}

We acknowledge partial support from the Department of Energy Office of Nuclear Physics under Contract No. DEFG02-96ER40989. We thank Mark Pitt for alerting us to the importance of parity-violating electron scattering in this context. We also thank Yu-Sheng Liu for his generous assistance with the exclusion plots and Heather Logan and Daniel Stolarski for prompting us to investigate the constraints from the running of $\alpha$ carefully. We thank Brian Batell, Bhupal Dev, and Tao Han for key input regarding the constraints from LEP on our model, and we thank Jeffrey Berryman and Yue Zhang for helpful discussions as well.

\section{APPENDIX: CALCULATIONAL DETAILS}

Herewith, we detail our $a_{\ell}$ computation for scalars that carry lepton number. The nature of the scalar-fermion interactions in this case, Eq. (5), allows for multiple ways in which the fermion fields can contract, so that it is more efficient to evaluate the time-ordered products of fields directly, rather than to develop Feynman rules for this case.

We have defined $\psi^{c}$ as $\psi^{c} \equiv C(\bar{\psi})^{\top}$, noting the charge conjugation matrix $C$ obeys

$$
\begin{aligned}
& \left.\overparen{\psi_{a}(x) \mid \boldsymbol{p}}, s\right\rangle=u_{a}(s, p) e^{-i p \cdot x}|0\rangle \quad\langle\boldsymbol{p}, s| \bar{\psi}_{a}(x)=\langle 0| \bar{u}_{a}(s, p) e^{i p \cdot x} \\
& \overline{\psi_{a}^{c}}(x)|\boldsymbol{p}, s\rangle=\overline{u_{a}^{c}}(s, p) e^{-i p \cdot x}|0\rangle \quad\left\langle\boldsymbol{p}, \overline{s \mid \psi_{a}^{c}}(x)=\langle 0| u_{a}^{c}(s, p) e^{i p \cdot x}\right.
\end{aligned}
$$$$
\begin{aligned}
\psi^{c}(x)= & \int \frac{d^{3} \boldsymbol{p}}{(2 \pi)^{3}} \frac{1}{\sqrt{2 E}} \\
& \times \sum_{s}\left(a_{\boldsymbol{p}}^{s \dagger} u^{c}(s, p) e^{i p \cdot x}+b_{\boldsymbol{p}}^{s} v^{c}(s, p) e^{-i p \cdot x}\right), \\
\overline{\psi^{c}}(x)= & \int \frac{d^{3} \boldsymbol{p}}{(2 \pi)^{3}} \frac{1}{\sqrt{2 E}} \\
& \times \sum_{s}\left(a_{\boldsymbol{p}}^{s} \overline{u^{c}}(s, p) e^{-i p \cdot x}+b_{\boldsymbol{p}}^{s \dagger} \overline{v^{c}}(s, p) e^{i p \cdot x}\right) .
\end{aligned}
$$

We note $u^{c}$ and $v^{c}$ are defined in the manner of $\psi^{c}$, and the creation and annihilation operators obey the anticommutation relations

$$
\left\{a_{\boldsymbol{p}}^{r}, a_{\boldsymbol{q}}^{s \dagger}\right\}=\left\{b_{\boldsymbol{p}}^{r}, b_{\boldsymbol{q}}^{s \dagger}\right\}=(2 \pi)^{3} \delta^{3}(\boldsymbol{p}-\boldsymbol{q}) \delta^{r s} .
$$

We now summarize all the Wick contractions that can appear. The contractions between $\psi(x), \bar{\psi}(x), \psi^{c}(x)$, and $\overline{\psi^{c}}(x)$ and an incoming or outgoing fermion of mass $m$ are

where $|\boldsymbol{p}, s\rangle=\sqrt{2 E_{p}} a_{p}^{s^{\dagger}}|0\rangle$ and $\langle\boldsymbol{p}, s|$ denote an incoming and an outgoing fermion with momentum $\boldsymbol{p}$ and spin $s$, respectively, whereas the contractions to an incoming or outgoing antifermion are

$$
\bar{\psi}_{a}(x)|\boldsymbol{k}, r\rangle=\bar{v}_{a}(r, k) e^{-i k \cdot x}|0\rangle \quad\langle\boldsymbol{k}, r| \psi_{a}(x)=\langle 0| v_{a}(r, p) e^{i k \cdot x}
$$




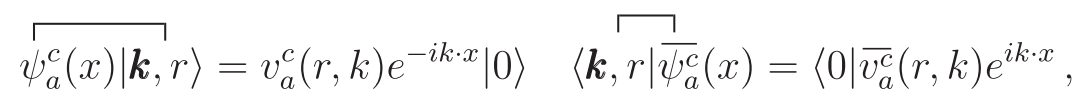

where, similarly, $|\boldsymbol{k}, r\rangle(\langle\boldsymbol{k}, r|)$ denote an incoming (outgoing) antifermion with momentum $\boldsymbol{k}$ and spin $r$. The spinor index $a$ runs from 1 to 4 . Different contractions of the internal fermion and antifermion fields can appear. That is,

$$
\begin{aligned}
& \overbrace{\psi_{a}(x)} \bar{\psi}_{b}(y)=\int \frac{d^{4} p}{(2 \pi)^{4}} \frac{i(\not p+m)_{a b}}{p^{2}-m^{2}+i \epsilon} e^{-i p \cdot(x-y)}, \\
& \overbrace{a}(x) \psi_{b}^{c}(y)=\int \frac{d^{4} p}{(2 \pi)^{4}} \frac{i\left[(\not p+m) C^{\top}\right]_{a b}}{p^{2}-m^{2}+i \epsilon} e^{-i p \cdot(x-y)} \\
& {\overline{\psi_{a}^{c}}}_{(x)} \bar{\psi}_{b}(y)=\int \frac{d^{4} p}{(2 \pi)^{4}} \frac{i\left[C^{\top}(\not p+m)\right]_{a b}}{p^{2}-m^{2}+i \epsilon} e^{-i p \cdot(x-y)},
\end{aligned}
$$

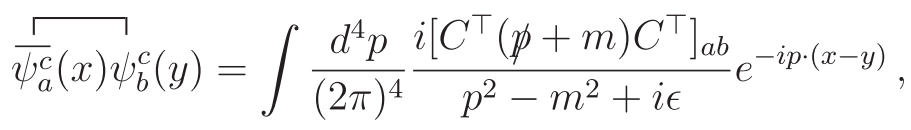

where $a$ and $b$ are spinor indices.

We now can compute the one-loop amplitude associated with the lepton anomalous magnetic dipole moment $a_{\ell}$. As shown in Fig. 1, a photon can be attached to either a charged fermion line or a charged scalar line, and interactions from QED and scalar QED are needed:

$$
\begin{gathered}
\mathcal{H}_{1} \supset-Q e \bar{\psi} \gamma^{\mu} \psi A_{\mu}, \\
\mathcal{H}_{2} \supset-i Q e\left[\left(\partial^{\mu} X\right) X^{*}-X\left(\partial^{\mu} X^{*}\right)\right] A_{\mu},
\end{gathered}
$$

where $Q=-1$ for the electron. Noting Eq. (5), we make the replacements $g_{i}^{11} \rightarrow g_{i}$ for $i=1,3$ and $e \rightarrow \psi$. Here, we consider the contributions from $X_{1}$ and $X_{3}^{3}$. We address the contribution to $a_{\ell}$ from $X_{2}$, as well as from $X_{3}^{2}$, later.

For the first case, the interaction is

$$
\mathcal{H} \supset-e Q \bar{\psi} \gamma^{\mu} \psi A_{\mu}+g_{i} X_{i} \overline{\psi^{c}} P_{\xi} \psi+g_{i}^{*} X_{i}^{*} \bar{\psi} P_{\xi^{\prime}} \psi^{c},
$$

where $P_{\xi}=\left(1+\xi \gamma^{5}\right) / 2$ is the chiral projection operator with $\xi= \pm 1$ for right (R) or left (L). Hermitian conjugation of the second term results in the third term, in which $\xi^{\prime}=-\xi$. The one-loop contribution comes from the $H^{3}$ term of the $S$ matrix,

$$
\left\langle\boldsymbol{p}^{\prime}\left|T\left(\frac{1}{3 !}(-i)^{3} \int d^{4} x \mathcal{H}(x) \int d^{4} y \mathcal{H}(y) \int d^{4} z \mathcal{H}(z)\right)\right| \boldsymbol{p} \boldsymbol{q}\right\rangle
$$

where $\boldsymbol{q}$ represent the momenta of incoming photon and $\boldsymbol{p}$ and $\boldsymbol{p}^{\prime}$ denote the momenta of the incoming and outgoing leptons, respectively. Since there are 3 ! ways of arranging the interactions in $\mathcal{H}$ to generate the same matrix element, we have

$$
\left\langle\boldsymbol{p}^{\prime}\left|T\left((-i)^{3} \int d^{4} x g_{i} X_{i} \overline{\psi^{c}} P_{\xi} \psi \int d^{4} y g_{i}^{*} X_{i}^{*} \bar{\psi} P_{\xi^{\prime}} \psi^{c} \int d^{4} z(-e Q) \bar{\psi} \gamma^{\mu} \psi A_{\mu}\right)\right| \boldsymbol{p} \boldsymbol{q}\right\rangle
$$

There are four different ways of contracting the fields in Eq. (A20), 


$$
\begin{aligned}
& \left\langle\boldsymbol{p}^{\prime}\left|\int d^{4} x X_{i} \overline{\psi_{a}^{c}}\left(P_{\xi}\right)_{a a^{\prime}} \psi_{a^{\prime}} \int d^{4} y X_{i}^{*} \overline{\psi_{b}}\left(P_{\xi^{\prime}}\right)_{b b^{\prime}} \psi_{b^{\prime}}^{c} \int d^{4} z \overline{\psi_{c}} \gamma_{c d}^{\mu} \psi_{d} A_{\mu}\right| \boldsymbol{p} \boldsymbol{q}\right\rangle, \\
& \left\langle\boldsymbol{p}^{\prime}\left|\int d^{4} x X_{i} \overline{\psi_{a}^{c}}\left(P_{\xi}\right)_{a a^{\prime}} \psi_{a^{\prime}} \int d^{4} y X_{i}^{*} \overline{\psi_{b}}\left(P_{\xi^{\prime}}\right)_{b b^{\prime}} \psi_{b^{\prime}}^{c} \int d^{4} z \overline{\psi_{c}} \gamma_{c d}^{\mu} \psi_{d} A_{\mu}\right| \boldsymbol{p} \boldsymbol{q}\right\rangle, \\
& \left\langle\boldsymbol{p}^{\prime}\left|\int d^{4} x X_{i} \overline{\psi_{a}^{c}}\left(P_{\xi}\right)_{a a^{\prime}} \psi_{a^{\prime}} \int d^{4} y X_{i}^{*} \overline{\psi_{b}}\left(P_{\xi^{\prime}}\right)_{b b^{\prime}} \psi_{b^{\prime}}^{c} \int d^{4} z \overline{\psi_{c}} \gamma_{c d}^{\mu} \psi_{d} A_{\mu}\right| \boldsymbol{p} \boldsymbol{q}\right\rangle, \\
& \left\langle\boldsymbol{p}^{\prime}\left|\int d^{4} x X_{i} \overline{\psi_{a}^{c}}\left(P_{\xi}\right)_{a a^{\prime}} \psi_{a^{\prime}} \int d^{4} y X_{i}^{*} \overline{\psi_{b}}\left(P_{\xi^{\prime}}\right)_{b b^{\prime}} \psi_{b^{\prime}}^{c} \int d^{4} z \overline{\psi_{c}} \gamma_{c d}^{\mu} \psi_{d} A_{\mu}\right| \boldsymbol{p} \boldsymbol{q}\right\rangle,
\end{aligned}
$$

where we have factored out $-(-i)^{3} g_{i} g_{i}^{*} Q e$ and have left the spinor indices explicit. After some manipulation, we find each contribution is identical; and after pulling out the factor $(2 \pi)^{4} \delta^{4}\left(p+q-p^{\prime}\right)$, the total matrix element is

$$
i \mathcal{M}^{\mu}=4 Q e\left|g_{i}\right|^{2} \int \frac{d^{4} k}{(2 \pi)^{4}} \frac{\bar{u}\left(p^{\prime}\right) P_{\xi^{\prime}}\left(\not k-m_{b}\right) \gamma^{\mu}\left(\not k^{\prime}-m_{b}\right) P_{\xi} u(p)}{\left(k^{2}-m_{b}^{2}+i \epsilon\right)\left(k^{\prime 2}-m_{b}^{2}+i \epsilon\right)\left(\left(k+p^{\prime}\right)^{2}-M_{X_{i}}^{2}+i \epsilon\right)},
$$

where $k^{\prime}=k+q$ and $m_{b}$ and $M_{X_{i}}$ are the masses of the charged lepton and scalar in the loop, respectively-the overall 4 comes from the different contractions we have noted. We find that Eq. (A31) contributes to $a_{\ell_{a}}$ as

$$
\delta a_{\ell_{a}}=\frac{Q g_{i} g_{i}^{*}}{4 \pi^{2}} \int_{0}^{1} d z \frac{m_{a}^{2} z(1-z)^{2}}{\left(z^{2}-z\right) m_{a}^{2}+z M_{X_{i}}^{2}+(1-z) m_{b}^{2}},
$$

where $m_{a}$ is the mass of external lepton a. Note that the final result is independent of $\xi$.

We now move to the second case. The interaction is

$$
\mathcal{H} \supset-i Q^{\prime} e\left[\left(\partial^{\mu} X_{i}\right) X_{i}^{*}-X_{i}\left(\partial^{\mu} X_{i}^{*}\right)\right] A_{\mu}+g_{i} X_{i} \bar{\psi}^{c} P_{\xi} \psi+g_{i}^{*} X_{i}^{*} \bar{\psi} P_{\xi^{\prime}} \psi^{c},
$$

where the charged scalar has $Q^{\prime}=2$, if it couples to two electrons. Here, too, there are four different contractions, and they contribute identically to the one-loop amplitude. Since there is only one way to contract all the scalars, we show it separately from the four different fermion contractions,

$$
\begin{aligned}
& \left\langle\boldsymbol{p}^{\prime}\left|\int d^{4} x \overline{\psi_{a}^{c}}\left(P_{\xi}\right)_{a a^{\prime}} \psi_{a^{\prime}} \int d^{4} y \overline{\psi_{b}}\left(P_{\xi^{\prime}}\right)_{b b^{\prime}} \psi_{b^{\prime}}^{c} \int d^{4} z A_{\mu}\right| \boldsymbol{p} \boldsymbol{q}\right\rangle, \\
& \left\langle\overline{\left.\boldsymbol{p}^{\prime}\left|\int d^{4} x \overline{\bar{\psi}_{a}^{c}}\left(P_{\xi}\right)_{a a^{\prime}} \psi_{a^{\prime}} \int d^{4} y \overline{\psi_{b}}\left(P_{\xi^{\prime}}\right)_{b b^{\prime}} \psi_{b^{\prime}}^{c} \int d^{4} z A_{\mu}\right| \boldsymbol{p} \boldsymbol{q}\right\rangle},\right.
\end{aligned}
$$




$$
\begin{gathered}
\left\langle\boldsymbol{p}^{\prime}\left|\int d^{4} x \overline{\psi_{a}^{c}}\left(P_{\xi}\right)_{a a^{\prime}} \psi_{a^{\prime}} \int d^{4} y \overline{\psi_{b}}\left(P_{\xi^{\prime}}\right)_{b b^{\prime}} \psi_{b^{\prime}}^{c} \int d^{4} z A_{\mu}\right| \boldsymbol{p} \boldsymbol{q}\right\rangle \\
\left\langle\stackrel{\left.\boldsymbol{p}^{\prime}\left|\int d^{4} x \overline{\psi_{a}^{c}}\left(P_{\xi}\right)_{a a^{\prime}} \psi_{a^{\prime}} \int d^{4} y \overline{\psi_{b}}\left(P_{\xi^{\prime}}\right)_{b b^{\prime}} \psi_{b^{\prime}}^{c} \int d^{4} z A_{\mu}\right| \boldsymbol{p} \boldsymbol{q}\right\rangle}{ },\right.
\end{gathered}
$$

with

$$
\left.i g_{i} g_{i}^{*} Q^{\prime} e[\overbrace{X_{i}(x) X_{i}^{*}(y)\left(i \partial^{\mu} X_{i}(z)\right) X_{i}^{*}}(z)-{ }_{X_{i}(x) X_{i}^{*}(y) X_{i}(z)\left(i \partial^{\mu} X_{i}^{*}\right.}(z))\right] .
$$

After combining all of the contractions and dropping the factor $(2 \pi)^{4} \delta^{4}\left(p+q-p^{\prime}\right)$, we find the one-loop matrix element in the second case is

$$
i \mathcal{M}^{\mu}=-4 g_{i} g_{i}^{*} Q^{\prime} e \int \frac{d^{4} k}{(2 \pi)^{4}} \frac{\bar{u}\left(p^{\prime}\right) P_{\xi^{\prime}}\left(\not k+\not k p^{\prime}+m_{b}\right) P_{\xi} u(p)\left(k+k^{\prime}\right)^{\mu}}{\left(k^{2}-M_{X_{i}}^{2}\right)\left(k^{\prime 2}-M_{X_{i}}^{2}\right)\left(\left(k+p^{\prime}\right)^{2}-m_{b}^{2}\right)}
$$

with $k^{\prime}=k+q$, which contributes to $a_{\ell_{a}}$ as

$\delta a_{\ell_{a}}=\frac{-Q^{\prime} g_{i} g_{i}^{*}}{4 \pi^{2}} \int_{0}^{1} d z \frac{m_{a}^{2} z(1-z)^{2}}{\left(z^{2}-z\right) m_{a}^{2}+z m_{b}^{2}+(1-z) M_{X_{i}}^{2}}$,

noting that this result is independent of $\xi$, too. To compute the final contribution to $\delta a_{\ell_{a}}$ from either $X_{1}$ or $X_{3}^{3}$ we add those of Eqs. (A26) and (A34).

The computation of $\delta a_{\ell_{e}}$ from $X_{2}$, or from $X_{3}^{2}$, is more straightforward in that only a single set of fermion contractions exists. We find from $X_{2}$, where $Q^{\prime}=1$ for the scalar that couples to an electron and a neutrino, that

$$
\delta a_{\ell_{a}}=\frac{-Q^{\prime} 4\left|g_{2}^{1 b}\right|^{2}}{16 \pi^{2}} \int_{0}^{1} d z \frac{m_{a}^{2} z(1-z)^{2}}{\left(z^{2}-z\right) m_{a}^{2}+z m_{b}^{2}+(1-z) M_{X_{i}}^{2}},
$$

where $m_{b}^{2}=m_{\nu_{b}}^{2}$. To find the contribution from $X_{3}^{2}$, we replace $2 g_{2}^{1 b}$ with $\sqrt{2} g_{3}^{11}$ and note that $m_{b}^{2}$ is just $m_{\nu_{e}}^{2}$.
[1] T. Aoyama, T. Kinoshita, and M. Nio, Theory of the anomalous magnetic moment of the electron, Atoms 7, 28 (2019).

[2] D. Hanneke, S. Fogwell, and G. Gabrielse, New Measurement of the Electron Magnetic Moment and the Fine Structure Constant, Phys. Rev. Lett. 100, 120801 (2008).

[3] R. Bouchendira, P. Cladé, S. Guellati-Khélifa, F. Nez, and F. Biraben, New Determination of the Fine Structure Constant and Test of the Quantum Electrodynamics, Phys. Rev. Lett. 106, 080801 (2011).

[4] R. H. Parker, C. Yu, W. Zhong, B. Estey, and H. Müller, Measurement of the fine-structure constant as a test of the standard model, Science 360, 191 (2018).

[5] G. W. Bennett et al. (Muon g-2 Collaboration), Final report of the Muon E821 anomalous magnetic moment measurement at BNL, Phys. Rev. D 73, 072003 (2006).
[6] B. L. Roberts and W. J. Marciano, Lepton Dipole Moments, Advanced Series on Directions in High Energy Physics (World Scientific, Singapore, 2009), Vol. 20, pp. 1-772, https://doi.org/10.1142/7273.

[7] M. Tanabashi et al. (Particle Data Group), Review of particle physics, Phys. Rev. D 98, 030001 (2018).

[8] P. J. Mohr, D. B. Newell, and B. N. Taylor, CODATA recommended values of the fundamental physical constants: 2014, Rev. Mod. Phys. 88, 035009 (2016).

[9] M. E. Peskin and D. V. Schroeder, An Introduction to Quantum Field Theory (Addison-Wesley, Reading, MA, 1995).

[10] M. Lindner, M. Platscher, and F. S. Queiroz, A call for new physics: The Muon anomalous magnetic moment and lepton flavor violation, Phys. Rep. 731, 1 (2018).

[11] T. Aoyama, M. Hayakawa, T. Kinoshita, and M. Nio, Tenth-Order QED Contribution to the Electron g-2 and an 
Improved Value of the Fine Structure Constant, Phys. Rev. Lett. 109, 111807 (2012).

[12] T. Aoyama, M. Hayakawa, T. Kinoshita, and M. Nio, Tenth-order electron anomalous magnetic momentContribution of diagrams without closed lepton loops, Phys. Rev. D 91, 033006 (2015); Erratum, Phys. Rev. D 96, 019901 (2017).

[13] S. Laporta, High-precision calculation of the 4-loop contribution to the electron g-2 in QED, Phys. Lett. B 772, 232 (2017).

[14] T. Aoyama, T. Kinoshita, and M. Nio, Revised and improved value of the QED tenth-order electron anomalous magnetic moment, Phys. Rev. D 97, 036001 (2018).

[15] A. D. Cronin, J. Schmiedmayer, and D. E. Pritchard, Optics and interferometry with atoms and molecules, Rev. Mod. Phys. 81, 1051 (2009).

[16] P. Cladé, F. Nez, F. Biraben, and S. Guellati-Khelifa, State of the art in the determination of the fine-structure constant and the ratio $h / m_{u}$, C. R. Phys. 20, 77 (2019).

[17] G. Gabrielse, S. E. Fayer, T. G. Myers, and X. Fan, Improved test of the standard model's most precise prediction, Atoms 7, 45 (2019).

[18] A. Czarnecki, B. Krause, and W. J. Marciano, Electroweak Corrections to the Muon Anomalous Magnetic Moment, Phys. Rev. Lett. 76, 3267 (1996).

[19] M. Knecht, S. Peris, M. Perrottet, and E. De Rafael, Electroweak hadronic contributions to the muon (g-2), J. High Energy Phys. 11 (2002) 003.

[20] A. Czarnecki, W. J. Marciano, and A. Vainshtein, Refinements in electroweak contributions to the muon anomalous magnetic moment, Phys. Rev. D 67, 073006 (2003); Erratum, Phys. Rev. D 73, 119901 (2006).

[21] D. Nomura and T. Teubner, Hadronic contributions to the anomalous magnetic moment of the electron and the hyperfine splitting of muonium, Nucl. Phys. B867, 236 (2013).

[22] F. Jegerlehner, Variations on photon vacuum polarization, EPJ Web Conf. 218, 01003 (2019).

[23] D. Hanneke, S. F. Hoogerheide, and G. Gabrielse, Cavity control of a single-electron quantum cyclotron: Measuring the electron magnetic moment, Phys. Rev. A 83, 052122 (2011).

[24] T. Blum, P. A. Boyle, V. Gülpers, T. Izubuchi, L. Jin, C. Jung, A. Jüttner, C. Lehner, A. Portelli, and J. T. Tsang (RBC and UKQCD Collaborations), Calculation of the Hadronic Vacuum Polarization Contribution to the Muon Anomalous Magnetic Moment, Phys. Rev. Lett. 121, 022003 (2018).

[25] A. Keshavarzi, D. Nomura, and T. Teubner, Muon $g-2$ and $\alpha\left(M_{Z}^{2}\right)$ : A new data-based analysis, Phys. Rev. D 97, 114025 (2018).

[26] G. F. Giudice, P. Paradisi, and M. Passera, Testing new physics with the electron g-2, J. High Energy Phys. 11 (2012) 113.

[27] J. Liu, C. E. M. Wagner, and X.-P. Wang, A light complex scalar for the electron and muon anomalous magnetic moments, J. High Energy Phys. 03 (2019) 008.

[28] H. Davoudiasl and W. J. Marciano, Tale of two anomalies, Phys. Rev. D 98, 075011 (2018).
[29] A. Crivellin, M. Hoferichter, and P. Schmidt-Wellenburg, Combined explanations of $(g-2)_{\mu, e}$ and implications for a large muon EDM, Phys. Rev. D 98, 113002 (2018).

[30] X.-F. Han, T. Li, L. Wang, and Y. Zhang, Simple interpretations of lepton anomalies in the lepton-specific inert two-Higgs-doublet model, Phys. Rev. D 99, 095034 (2019).

[31] P. Fayet, U-boson production in $e+e-$ annihilations, psi and Upsilon decays, and light dark matter, Phys. Rev. D 75, 115017 (2007).

[32] M. Pospelov, Secluded U(1) below the weak scale, Phys. Rev. D 80, 095002 (2009).

[33] N. Arkani-Hamed, D. P. Finkbeiner, T. R. Slatyer, and N. Weiner, A theory of dark matter, Phys. Rev. D 79, 015014 (2009).

[34] J. D. Bjorken, R. Essig, P. Schuster, and N. Toro, New fixed-target experiments to search for dark gauge forces, Phys. Rev. D 80, 075018 (2009).

[35] B. Batell, M. Pospelov, and A. Ritz, Exploring portals to a hidden sector through fixed targets, Phys. Rev. D 80, 095024 (2009).

[36] R. Essig et al., Dark sectors and new, light weakly coupled particles, in Proceedings, 2013 Community Summer Study on the Future of U.S. Particle Physics: Snowmass on the Mississippi (CSS2013) (2013), https://www.slac.stanford .edu/econf/C1307292/.

[37] J. Alexander et al., Dark sectors 2016 workshop: Community report (2016), https://arxiv.org/pdf/1608 .08632.pdf.

[38] B. Holdom, Two U(1)'s and Epsilon charge shifts, Phys. Lett. 166B, 196 (1986).

[39] J. P. Lees et al. (BABAR Collaboration), Search for Invisible Decays of a Dark Photon Produced in $e^{+} e^{-}$ Collisions at BABAR, Phys. Rev. Lett. 119, 131804 (2017).

[40] C.-Y. Chen, H. Davoudiasl, W. J. Marciano, and C. Zhang, Implications of a light 'dark Higgs' solution to the $g_{\mu}-2$ discrepancy, Phys. Rev. D 93, 035006 (2016).

[41] Y.-S. Liu, D. McKeen, and G. A. Miller, Electrophobic Scalar Boson and Muonic Puzzles, Phys. Rev. Lett. 117, 101801 (2016).

[42] S. M. Barr and A. Zee, Electric Dipole Moment of the Electron and of the Neutron, Phys. Rev. Lett. 65, 21 (1990); Erratum, Phys. Rev. Lett. 65, 2920 (1990).

[43] T. Kinoshita and W. J. Marciano, Theory of the muon anomalous magnetic moment, Adv. Ser. Dir. High Energy Phys. 7, 419 (1990).

[44] V. Barger, C.-W. Chiang, W.-Y. Keung, and D. Marfatia, Proton Size Anomaly, Phys. Rev. Lett. 106, 153001 (2011).

[45] D. Tucker-Smith and I. Yavin, Muonic hydrogen and MeV forces, Phys. Rev. D 83, 101702 (2011).

[46] B. Batell, N. Lange, D. McKeen, M. Pospelov, and A. Ritz, Muon anomalous magnetic moment through the leptonic Higgs portal, Phys. Rev. D 95, 075003 (2017).

[47] A. J. Davies and X.-G. He, Tree level scalar Fermion interactions consistent with the symmetries of the standard model, Phys. Rev. D 43, 225 (1991).

[48] J. P. Bowes, R. Foot, and R. R. Volkas, Electric charge quantization from gauge invariance of a Lagrangian: A 
catalog of baryon number violating scalar interactions, Phys. Rev. D 54, 6936 (1996).

[49] J. M. Arnold, B. Fornal, and M. B. Wise, Simplified models with baryon number violation but no proton decay, Phys. Rev. D 87, 075004 (2013).

[50] J. M. Arnold, B. Fornal, and M. B. Wise, Phenomenology of scalar leptoquarks, Phys. Rev. D 88, 035009 (2013).

[51] S. Gardner and X. Yan, Processes that break baryon number by two units and the Majorana nature of the neutrino, Phys. Lett. B 790, 421 (2019).

[52] K. Kannike, M. Raidal, D. M. Straub, and A. Strumia, Anthropic solution to the magnetic muon anomaly: the charged see-saw, J. High Energy Phys. 02 (2012) 106; Erratum, J. High Energy Phys. 10 (2012) 136.

[53] J. Girrbach, S. Mertens, U. Nierste, and S. Wiesenfeldt, Lepton flavour violation in the MSSM, J. High Energy Phys. 05 (2010) 026.

[54] B. Dutta and Y. Mimura, Electron $g-2$ with flavor violation in MSSM, Phys. Lett. B 790, 563 (2019).

[55] Y. Kahn, G. Krnjaic, S. Mishra-Sharma, and T. M. P. Tait, Light weakly coupled axial forces: Models, constraints, and projections, J. High Energy Phys. 05 (2017) 002.

[56] P. Minkowski, $\mu \rightarrow e \gamma$ at a rate of one out of $10^{9}$ muon decays?, Phys. Lett. 67B, 421 (1977).

[57] M. Gell-Mann, P. Ramond, and R. Slansky, Complex spinors and unified theories, Conf. Proc. C 790927, 315 (1979), https://arxiv.org/pdf/1306.4669.pdf.

[58] T. Yanagida, in 60 Years of Double Beta Decay from Nuclear Physics to Beyond Standard Model Particle Physics, edited by H. V. Klapdor-Kleingrothaus (World Scientific, Singapore, 2001), p. 169.

[59] R. N. Mohapatra and G. Senjanovic, Neutrino Mass and Spontaneous Parity Nonconservation, Phys. Rev. Lett. 44, 912 (1980).

[60] A. de Gouvêa, Neutrino mass models, Annu. Rev. Nucl. Part. Sci. 66, 197 (2016).

[61] W. Konetschny and W. Kummer, Nonconservation of total lepton number with scalar bosons, Phys. Lett. 70B, 433 (1977).

[62] M. Magg and C. Wetterich, Neutrino mass problem and gauge hierarchy, Phys. Lett. 94B, 61 (1980).

[63] J. Schechter and J.W.F. Valle, Neutrino masses in $\mathrm{SU}(2) \times \mathrm{U}(1)$ theories, Phys. Rev. D 22, 2227 (1980).

[64] T. P. Cheng and L.-F. Li, Neutrino Masses, mixings and oscillations in $\mathrm{SU}(2) \times \mathrm{U}(1)$ models of electroweak interactions, Phys. Rev. D 22, 2860 (1980).

[65] R. N. Mohapatra and G. Senjanovic, Neutrino masses and mixings in gauge models with spontaneous parity violation, Phys. Rev. D 23, 165 (1981).

[66] A. Zee, A theory of lepton number violation, neutrino Majorana mass, and oscillation, Phys. Lett. 93B, 389 (1980); Erratum, Phys. Lett. 95B, 461 (1980).

[67] A. Zee, Quantum numbers of Majorana neutrino masses, Nucl. Phys. B264, 99 (1986).

[68] K. S. Babu, Model of "Calculable" Majorana neutrino masses, Phys. Lett. B 203, 132 (1988).

[69] L. J. Hall and M. Suzuki, Explicit R-parity breaking in supersymmetric models, Nucl. Phys. B231, 419 (1984).

[70] D. Chang and R. N. Mohapatra, Small and Calculable Dirac Neutrino Mass, Phys. Rev. Lett. 58, 1600 (1987).
[71] K. S. Babu and C. N. Leung, Classification of effective neutrino mass operators, Nucl. Phys. B619, 667 (2001).

[72] K. S. Babu and C. Macesanu, Two loop neutrino mass generation and its experimental consequences, Phys. Rev. D 67, 073010 (2003).

[73] Y. Cai, J. Herrero-García, M. A. Schmidt, A. Vicente, and R. R. Volkas, From the trees to the forest: A review of radiative neutrino mass models, Front. Phys. 5, 63 (2017).

[74] P. S. B. Dev, M. J. Ramsey-Musolf, and Y. Zhang, Doublycharged scalars in the type-II Seesaw mechanism: Fundamental symmetry tests and high-energy searches, Phys. Rev. D 98, 055013 (2018).

[75] S. M. Barr and X. Calmet, Observable proton decay from planck scale physics, Phys. Rev. D 86, 116010 (2012).

[76] R. N. Mohapatra and R. E. Marshak, Local B-L symmetry of electroweak interactions, Majorana neutrinos and neutron oscillations, Phys. Rev. Lett. 44, 1316 (1980); Erratum, Phys. Rev. Lett. 44, 1643 (1980).

[77] K. S. Babu and R. N. Mohapatra, Observable neutron antineutron oscillations in Seesaw models of neutrino mass, Phys. Lett. B 518, 269 (2001).

[78] Z. Chacko and R. Mohapatra, Supersymmetric SU(2)-L x SU(2)-R x SU(4)-c and observable neutron-anti-neutron oscillation, Phys. Rev. D 59, 055004 (1999).

[79] K. S. Babu, P. S. Bhupal Dev, and R. N. Mohapatra, Neutrino mass hierarchy, neutron-anti-neutron oscillation from baryogenesis, Phys. Rev. D 79, 015017 (2009).

[80] K. Babu and R. Mohapatra, Coupling unification, GUTscale baryogenesis and neutron-antineutron oscillation in SO(10), Phys. Lett. B 715, 328 (2012).

[81] H. K. Dreiner, H.E. Haber, and S.P. Martin, Twocomponent spinor techniques and Feynman rules for quantum field theory and supersymmetry, Phys. Rep. 494, 1 (2010).

[82] S. Gardner and X. Yan, $C P T, C P$, and $\mathrm{C}$ transformations of Fermions, and their consequences, in theories with B-L violation, Phys. Rev. D 93, 096008 (2016).

[83] S. Knapen, T. Lin, and K. M. Zurek, Light dark matter: Models and constraints, Phys. Rev. D 96, 115021 (2017).

[84] E. Rrapaj and S. Reddy, Nucleon-nucleon bremsstrahlung of dark gauge bosons and revised supernova constraints, Phys. Rev. C 94, 045805 (2016).

[85] S. Chatrchyan et al. (CMS Collaboration), Search for new physics in events with same-sign dileptons and jets in pp collisions at $\sqrt{s}=8 \mathrm{TeV}$, J. High Energy Phys. 01 (2014) 163; 01 (2015) 14.

[86] V. Khachatryan et al. (CMS Collaboration), Search for new physics in same-sign dilepton events in proton-proton collisions at $\sqrt{s}=13 \mathrm{TeV}$, Eur. Phys. J. C 76, 439 (2016).

[87] P. L. Anthony et al. (SLAC E158 Collaboration), Precision Measurement of the Weak Mixing Angle in Moller Scattering, Phys. Rev. Lett. 95, 081601 (2005).

[88] Moller: Jefferson lab experiment e12-09-005, https:// hallaweb.jlab.org/12GeV/Moller/pubs/moller_proposal.pdf.

[89] J. Benesch et al. (MOLLER Collaboration), The MOLLER experiment: An ultra-precise measurement of the weak mixing angle using Møller scattering, arXiv:1411.4088.

[90] J. Abdallah et al. (DELPHI Collaboration), Measurement and interpretation of fermion-pair production at LEP 
energies above the $\mathrm{Z}$ resonance, Eur. Phys. J. C 45, 589 (2006).

[91] E. Derman and W. J. Marciano, Parity violating asymmetries in polarized electron scattering, Ann. Phys. (N.Y.) 121, 147 (1979).

[92] A. Czarnecki and W. J. Marciano, Electroweak radiative corrections to polarized Moller scattering asymmetries, Phys. Rev. D 53, 1066 (1996).

[93] A. Ferroglia, G. Ossola, and A. Sirlin, The electroweak form-factor $\hat{\kappa}\left(q^{2}\right)$ and the running of $\sin ^{2} \hat{\theta}_{\mathrm{W}}$, Eur. Phys. J. C 34, 165 (2004).

[94] J. Erler, A. Kurylov, and M. J. Ramsey-Musolf, The weak charge of the proton and new physics, Phys. Rev. D 68 , 016006 (2003).

[95] J. Erler and M. J. Ramsey-Musolf, The weak mixing angle at low energies, Phys. Rev. D 72, 073003 (2005).

[96] F. J. Petriello, Radiative corrections to fixed target Moller scattering including hard bremsstrahlung effects, Phys. Rev. D 67, 033006 (2003).

[97] E. Eichten, K. D. Lane, and M. E. Peskin, New Tests for Quark and Lepton Substructure, Phys. Rev. Lett. 50, 811 (1983).

[98] C. C. Nishi, Simple derivation of general Fierz-like identities, Am.J. Phys. 73, 1160 (2005).

[99] M. L. Swartz, Limits on doubly charged Higgs bosons and lepton flavor violation, Phys. Rev. D 40, 1521 (1989).

[100] Y.-S. Liu, D. McKeen, and G. A. Miller, Validity of the Weizsäcker-Williams approximation and the analysis of beam dump experiments: Production of a new scalar boson, Phys. Rev. D 95, 036010 (2017).

[101] H. Tsertos, C. Kozhuharov, P. Armbruster, P. Kienle, B. Krusche, and K. Schreckenbach, High sensitivity measurements of the excitation function for Bhabha scattering at MeV energies, Phys. Rev. D 40, 1397 (1989).

[102] A. G. Beda, V. B. Brudanin, V. G. Egorov, D. V. Medvedev, V. S. Pogosov, M. V. Shirchenko, and A. S. Starostin, The results of search for the neutrino magnetic moment in GEMMA experiment, Adv. High Energy Phys. 2012, 350150 (2012).

[103] C. Giunti, K. A. Kouzakov, Y.-F. Li, A. V. Lokhov, A. I. Studenikin, and S. Zhou, Electromagnetic neutrinos in laboratory experiments and astrophysics, Ann. Phys. (Amsterdam) 528, 198 (2016).

[104] A. Studenikin, Status and perspectives of neutrino magnetic moments, J. Phys. Conf. Ser. 718, 062076 (2016).

[105] S. Gardner, R. J. Holt, and A. S. Tadepalli, New prospects in fixed target searches for dark forces with the
SeaQuest experiment at Fermilab, Phys. Rev. D 93, 115015 (2016).

[106] A. Pich, Precision Tau physics, Prog. Part. Nucl. Phys. 75, 41 (2014).

[107] G. Gelmini and M. Roncadelli, Left-handed neutrino mass scale and spontaneously broken lepton number, Phys. Lett. 99B, 411 (1981).

[108] R. N. Mohapatra and J. D. Vergados, A New Contribution to Neutrinoless Double Beta Decay in Gauge Models, Phys. Rev. Lett. 47, 1713 (1981).

[109] V. D. Barger, H. Baer, W.-Y. Keung, and R. Phillips, Decays of weak vector bosons and T quarks into doubly charged Higgs scalars, Phys. Rev. D 26, 218 (1982).

[110] J. Grifols, A. Mendez, and G. Schuler, Production and decay of doubly charged Higgs bosons of left-right symmetric models, Mod. Phys. Lett. A 04, 1485 (1989).

[111] M. Aaboud et al. (ATLAS Collaboration), Search for doubly charged Higgs boson production in multi-lepton final states with the ATLAS detector using proton-proton collisions at $\sqrt{s}=13 \mathrm{TeV}$, Eur. Phys. J. C 78, 199 (2018).

[112] CMS Collaboration, CERN Report No. CMS-PAS-HIG16-036, 2017.

[113] G. Abbiendi et al. (ALEPH, DELPHI, L3, OPAL, and LEP Collaboration), Search for charged Higgs bosons: Combined results using LEP data, Eur. Phys. J. C 73, 2463 (2013).

[114] G. Abbiendi et al. (OPAL Collaboration), Search for the single production of doubly charged Higgs bosons and constraints on their couplings from Bhabha scattering, Phys. Lett. B 577, 93 (2003).

[115] G. Abbiendi et al. (OPAL Collaboration), Search for doubly charged Higgs bosons with the OPAL detector at LEP, Phys. Lett. B 526, 221 (2002).

[116] P. Acton et al. (OPAL Collaboration), A search for doubly charged Higgs production in Z0 decays, Phys. Lett. B 295, 347 (1992).

[117] F. Le Diberder, Study of the annihilation reactions $e^{+} e^{-}$ into four leptons, Ph.D. thesis, Orsay LAL, 1988, https:// inis.iaea.org/collection/NCLCollectionStore_Public/20/ 049/20049964.pdf.

[118] A. Anastasi et al. (KLOE-2 Collaboration), Measurement of the running of the fine structure constant below $1 \mathrm{GeV}$ with the KLOE Detector, Phys. Lett. B 767, 485 (2017).

[119] F. Jegerlehner, Hadronic contributions to the photon vacuum polarization and their role in precision physics, J. Phys. G 29, 101 (2003).

[120] R. S. Van Dyck, P. B. Schwinberg, and H. G. Dehmelt, New High-Precision Comparison of Electron and Positron g Factors, Phys. Rev. Lett. 59, 26 (1987). 